\title{
On Eigenstructure-Based Direct Multichannel Blind Image Restoration
}

\author{
Hung-Ta Pai, Member, IEEE, and Alan Conrad Bovik, Fellow, IEEE
}

\begin{abstract}
Existing eigenstructure-based direct multichannel blind image restoration techniques include nullspace-based and direct deconvolver estimation techniques. The nullspace-based approach can be formulated as an optimization problem. We show that this formulation implies a new subspace-based approach that uses matrix operations. This new approach has the same advantages as the nullspace-based one but requires less computational complexity. Under some mild conditions, its complexity is equal to that of the FFT. Furthermore, the relation among the nullspace-based approach, the direct deconvolver estimation and the new subspace-based approach is studied.
\end{abstract}

Index Terms-Blind deconvolution, image restoration, nullspace, subspace.

\section{INTRODUCTION}

$\mathbf{I}$ N MANY applications such as medical imaging, radio astronomy and remote sensing, the observed images are degraded by distortion. Distortion may arise from, for example, atmospheric turbulence, relative motion between an object and the camera, an out-of-focus camera, or variations in electronic imaging components. Restoration of the degraded images is generally desirable for further processing or interpretation of the images [1].

To restore the original image, a model of the original image and a degradation model are first assumed. The original image may also be regarded as either a deterministic or stochastic signal. It is blurred by a linear or nonlinear process. An additive or multiplicative noise process may be generated in the acquisition of the images. Because constraints on the degradation and the original image vary with the application, many different algorithms exist.

Many conventional approaches have been developed to compensate for the blur function when it is known [2]. More commonly, however, the blur function is unknown. It is not practical to assume the availability of training images either. In such cases, a model of the blur is often assumed, for instance, a linear space-invariant filter. When the blur is unknown, the problem is called blind image restoration. It is a very difficult problem since there are two unknowns, the original image and the blur and only one equation, the blur model.

Manuscript received February 29, 2000; revised June 25, 2001. The associate editor coordinating the review of this manuscript and approving it for publication was Prof. Timothy J. Schulz.

The authors are with the Laboratory for Image and Video Engineering (LIVE), Department of Electrical and Computer Engineering, The University of Texas, Austin, TX 78712-1084 USA (e-mail: pai@vision.ece.utexas.edu; bovik@ece.utexas.edu).

Publisher Item Identifier S 1057-7149(01)08205-7.
There are two classes of approaches to blind image restoration. One class identifies the blur first and then uses it to restore the original image with one of the conventional image restoration algorithms. Algorithms in this class make more assumptions on the original image, for instance, that the image consists of point sources and edges. Therefore, their applications are limited. The other class simultaneously identifies the blur and restores the original image. All methods in this class are either iterative or recursive. Typically, they suffer from convergence and/or stability problems. A review article [3] and an updated version [4] for completeness on this topic are available. Sometimes the blur function is only partially determined, as in [5].

In some applications, several blurred versions of the same original image are available from different blurring channels, for instance, in short-exposure image sequences. Restoring the original image in this scenario is called multichannel blind image restoration. Two classes of multichannel blind image restoration algorithms exist. One class includes extensions of single-channel blind image restoration approaches [6], [7]. The algorithms in this class have the same problems, such as convergence and stability problems, as their single-channel counterparts [3], [4]. The other class encompasses extensions of blind multichannel one-dimensional (1-D) signal estimation methods.

Recently, Tong et al. [8], [9] proposed a novel algorithm to estimate blind 1-D communication channels. They assumed that signals are deterministic, the channels are linear spatial invariant (LSI) finite impulse response (FIR) functions with known length and that the noise is additive. They oversampled the received 1-D communication signal temporally, spatially, or both temporally and spatially. The oversampled signal could be modeled as the output of a multichannel system driven by the same signal. Based on the second-order statistics of the oversampled signal, the channel was estimated using algebraic techniques. In noise-free cases, the channels could be exactly recovered, up to a scalar multiplier. In noisy cases, the algorithm also obtained very promising results. The original signal was extracted by a classical signal estimation algorithm, for instance, using Wiener filtering based on the estimated channel.

Several approaches, which followed this idea, for multichannel 1-D blind signal deconvolution have been proposed to identify the channel [10]-[12]. One of them is the so-called eigenstructure-based (ESB) method. It includes subspace [13], [14] and least-squares [15], [16] approaches. The subspace approach uses properties of subspaces of a special matrix constructed by the channel impulse responses. The least-squares approach exploits the property of a multichannel system that has one input and multiple outputs. In other words, the estimate 
of the input from one of the outputs should be equal to the estimate from another output.

Based on the Bezout identity for coprime polynomials [17], Slock et al. [13], [18] proposed an algorithm to estimate equalizers. The channel and the equalizer could be considered as an analysis and synthesis filter bank. By convolving the degraded image with the equalizer, the original image could be estimated. In [19], using ideas similar to the least-squares approach in the channel estimation, a direct estimation of the equalizer was proposed.

By extending the least-squares approach and the algorithm of Slock et al. to two dimensions, Harikumar et al. [20], [21] developed an algorithm for multichannel blind image restoration. The algorithm estimated the blur functions first and/or find restoration filters (deconvolver). The original image was restored using conventional image restoration methods or by convolving the observed image with the restoration filter. Giannakis et al. [22] proposed two algorithms at about the same time. One algorithm is similar to the algorithm of Harikumar et $a l$. The other is a direct deconvolver estimation algorithm which is an extension of the direct equalizer estimation algorithm [19]. In the noise-free case, the algorithms developed by Harikumar et al. and Giannakis et al. can obtain the orignal image, up to a scalar multiplier, as their 1-D counterparts. However, in the noisy case, the obtained image suffers from noise amplification.

Instead of identifying the channel or estimating the equalizer, an algorithm was proposed to estimate the original 1-D signal directly in [23]. It employed the null space of a special matrix constructed by the original signal. Another algorithm was then proposed by exploiting the column space of the special matrix [24]. The estimated signals of both algorithms are the same. In [25], the variants of these two algorithms and the direct equalizer estimation algorithm were shown to be equivalent. In addition, these two algorithms result in better signal estimation than the channel identification algorithms.

In [26]-[28], using the null space approach, Pai et al. proposed an algorithm for direct multichannel blind image restoration. This algorithm and the direct deconvolver estimation algorithm are direct multichannel blind image restoration algorithms because they do not require other algorithms to estimate the original image. In the noise-free case, the original image can be exactly restored, up to a scalar ambiguity. On the other hand, there is no noise amplification in the noisy case.

In this paper, we first pose the restoration problem in noisy cases using the null space as a variety of nonlinear optimization problems with different constraints. By properly choosing the constraints, these optimization problems can be solved using matrix operations. Moreover, in the noise-free case, their solutions are equal to the original image. We then formulate a new constrained optimization problem for image restoration using the column space. This new problem is similar to but different from the one in [24]. A new algorithm using matrix operations to solve the new problem is proposed and its implementation issues are discussed. Secondly, we compare the new algorithm with the direct deconvolver estimation algorithm by techniques similar to [25]. The direct deconvolver estimation algorithm can also be formulated as a constrained optimization problem. Using a different constraint and a different weighting on the object function of the constrained optimization problem, the direct deconvolver estimation algorithm is equal to the new algorithm.

The remainder of this paper is organized as follows. Section II describes a model for a multichannel imaging system and defines notation. In Section III, we address the different optimization problems and propose a new algorithm. The implementation issues of the new algorithm are discussed in Section IV. Section V derives the relation between the new algorithm and the direct deconvolver estimation algorithm. Simulation results using the new algorithm are reported in Section VI. Finally, we briefly conclude our contributions in Section VII.

\section{Problem Statement}

As shown in Fig. 1, the output of the $i$ th channel in an $m$-channel FIR LTI image system is given by

$$
x^{(i)}=h^{(i)} * s+e^{(i)}
$$

$\begin{array}{cl}\text { where } & \\ x^{(i)} & \text { ith observed }\left(n_{1}-l_{1}+1\right) \times\left(n_{2}-l_{2}+1\right) \text { degraded } \\ & \text { image; } \\ h^{(i)} & i \text { th } l_{1} \times l_{2} \text { blur function; } \\ s & n_{1} \times n_{2} \text { original image; } \\ e^{(i)} & \text { additive noise. }\end{array}$ For convenience, let $x^{(i)}, h^{(i)}, s$ and $e^{(i)}$ be indexed from $\left(l_{1}-\right.$ $\left.1, l_{2}-1\right)$ to $\left(n_{1}-1, n_{2}-1\right)$, from $(0,0)$ to $\left(l_{1}-1, l_{2}-1\right)$, from $(0,0)$ to $\left(n_{1}-1, n_{2}-1\right)$ and from $\left(l_{1}-1, l_{2}-1\right)$ to $\left(n_{1}-1, n_{2}-1\right)$, respectively.

In this paper, all lowercase characters are scalars. All vectors are column vectors and denoted by boldface lowercase characters. Uppercase and boldface uppercase characters denote matrices. The largest/smallest eigenvalue means the eigenvalue of the largest/smallest magnitude. Other notation used is as follows:
$(\cdot)^{T}$
$\|\cdot\|$
transpose;
I Frobenius norm; identity matrix;
$\operatorname{diag}\{\mathbf{A}\} \quad$ main diagonal of $\mathbf{A}$
$\mathcal{N}\{\mathbf{A}\}$ null space of $\mathbf{A}$
$\mathcal{R}\{\mathbf{A}\}$ range of $\mathbf{A}$ (i.e., column space of $\mathbf{A})$.

We also abuse notation and write $\mathcal{N}\{\mathbf{A}\} / \mathcal{R}\{\mathbf{A}\}$ to mean a matrix whose columns form an orthonormal basis for the null/column space of $\mathbf{A}$

\section{SUBSPACE APPROACHES}

\section{A. Review of the Null Space Approach}

As in [26]-[28], define (see the equation at the bottom of the next page) where $k_{1}$ and $k_{2}$ are numbers of shifts in $\mathbf{X}$ and $X_{p_{1}}$, respectively. The matrices defined above are formed to represent the convolution operation in (1). The sizes of $\mathbf{X}, \mathbf{H}$ and $\mathbf{S}$ are determined by $k_{1}$ and $k_{2}$. Therefore, in the noise-free case

$$
\mathbf{X}=\mathbf{H S} \text {. }
$$

Define $r_{1}=l_{1}+k_{1}-2$ and $r_{2}=l_{2}+k_{2}-2$. Notice that the matrices $\mathbf{X}, \mathbf{H}$ and $\mathbf{S}$ are $m k_{1} k_{2} \times\left(n_{1}-r_{1}\right)\left(n_{2}-r_{2}\right)$, $m k_{1} k_{2} \times\left(r_{1}+1\right)\left(r_{2}+1\right)$ and $\left(r_{1}+1\right)\left(r_{2}+1\right) \times\left(n_{1}-r_{1}\right)\left(n_{2}-r_{2}\right)$ matrices, respectively. If $\mathbf{H}$ has full column rank by properly 


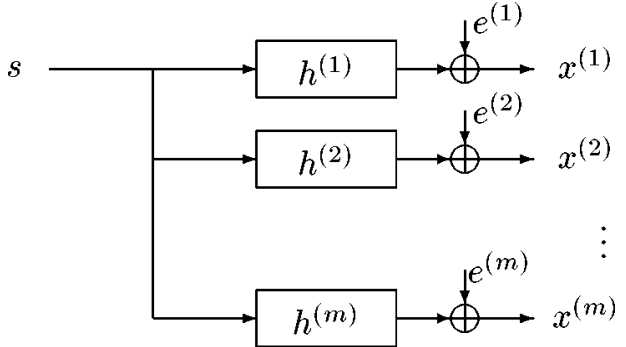

choosing $k_{1}$ and $k_{2}$, then $\mathcal{N}\{\mathbf{X}\}=\mathcal{N}\{\mathbf{S}\}$. Notice that $\mathcal{N}\{\mathbf{S}\}$ is an $\left(\left(n_{1}-r_{1}\right)\left(n_{2}-r_{2}\right)-\left(r_{1}+1\right)\left(r_{2}+1\right)\right) \times\left(n_{1}-r_{1}\right)\left(n_{2}-\right.$ $\left.r_{2}\right)$ matrix. Let $v_{q_{1}, q_{2}}$ and $v_{q_{1}, q_{2}}^{\prime}$ be the $\left(q_{1}, q_{2}\right)$ entry of $\mathcal{N}\{\mathbf{S}\}$ and $\mathcal{N}\{\mathbf{X}\}$ respectively. Define (see the equation shown at the bottom of the next page). Notice that the matrix $\mathbf{V}$ is an $\left(\left(n_{1}-\right.\right.$ $\left.\left.r_{1}\right)\left(n_{2}-r_{2}\right)-\left(r_{1}+1\right)\left(r_{2}+1\right)\right) r_{1} r_{2} \times n_{1} n_{2}$ matrix. $\mathbf{v}_{p_{1}, p_{2}}^{\prime}$, $V_{p_{1}}^{\prime}$ and $\mathbf{V}^{\prime}$ are defined with $v_{q_{1}, q_{2}}^{\prime}$ as $\mathbf{v}_{p_{1}, p_{2}}, V_{p_{1}}$ and $\mathbf{V}$ with $v_{q_{1}, q_{2}}$. In the noise-free case, $\mathcal{R}\left\{\mathbf{V}^{\prime}\right\}=\mathcal{R}\{\mathbf{V}\}$. Thus,

Fig. 1. Single-input multiple-output image-blur model. The blurring functions $h^{(1)}, h^{(2)}, \ldots, h^{(m)}$ are assumed to be LTI FIR filters.

$$
\mathrm{Vs}=\mathbf{0}
$$

$$
\begin{aligned}
& \mathbf{x}_{p_{1}, p_{2}}=\left[x_{p_{1}, p_{2}}^{(1)}, x_{p_{1}, p_{2}}^{(2)}, \ldots, x_{p_{1}, p_{2}}^{(m)}\right]^{T} \\
& X_{p_{1}}=\underbrace{\left[\begin{array}{cccc}
\mathbf{x}_{p_{1}, l_{2}-1} & \mathbf{x}_{p_{1}, l_{2}} & \cdots & \mathbf{x}_{p_{1}, n_{2}-k_{2}} \\
\mathbf{x}_{p_{1}, l_{2}} & \mathbf{x}_{p_{1}, l_{2}+1} & \cdots & \mathbf{x}_{p_{1}, n_{2}-k_{2}+1} \\
\vdots & \vdots & \vdots & \vdots \\
\mathbf{x}_{p_{1}, l_{2}+k_{2}-2} & \mathbf{x}_{p_{1}, l_{2}+k_{2}-1} & \cdots & \mathbf{x}_{p_{1}, n_{2}-1}
\end{array}\right]}_{n_{2}-l_{2}-k_{2}+2 \text { blocks }} \\
& \mathbf{X}=\underbrace{\left[\begin{array}{cccc}
X_{l_{1}-1} & X_{l_{1}} & \cdots & X_{n_{1}-k_{1}} \\
X_{l_{1}} & X_{l_{1}+1} & \cdots & X_{n_{1}-k_{1}+1} \\
\vdots & \vdots & \vdots & \vdots \\
X_{l_{1}+k_{1}-2} & X_{l_{1}+k_{1}-1} & \cdots & X_{n_{1}-1}
\end{array}\right]}_{n_{1}-l_{1}-k_{1}+2 \text { blocks }} \\
& \mathbf{h}_{p_{1}, p_{2}}=\left[h_{p_{1}, p_{2}}^{(1)}, h_{p_{1}, p_{2}}^{(2)}, \ldots, h_{p_{1}, p_{2}}^{(m)}\right]^{T} \\
& H_{p_{1}}=\underbrace{\left[\begin{array}{ccccccc}
\mathbf{h}_{p_{1}, l_{2}-1} & \mathbf{h}_{p_{1}, l_{2}-2} & \cdots & \mathbf{h}_{p_{1}, 0} & \mathbf{0} & \cdots & \mathbf{0} \\
\mathbf{0} & \mathbf{h}_{p_{1}, l_{2}-1} & \mathbf{h}_{p_{1}, l_{2}-2} & \cdots & \mathbf{h}_{p_{1}, 0} & \ddots & \mathbf{0} \\
\vdots & \ddots & \ddots & \ddots & \ddots & \ddots & \vdots \\
\mathbf{0} & \cdots & \mathbf{0} & \mathbf{h}_{p_{1}, l_{2}-1} & \mathbf{h}_{p_{1}, l_{2}-2} & \cdots & \mathbf{h}_{p_{1}, 0}
\end{array}\right]}_{l_{2}+k_{2}-1 \text { blocks }} \\
& \mathbf{H}=\underbrace{\left[\begin{array}{ccccccc}
H_{l_{1}-1} & H_{l_{1}-2} & \cdots & H_{0} & \mathbf{0} & \cdots & \mathbf{0} \\
\mathbf{0} & H_{l_{1}-1} & H_{l_{1}-2} & \cdots & H_{0} & \ddots & \mathbf{0} \\
\vdots & \ddots & \ddots & \ddots & \ddots & \ddots & \vdots \\
\mathbf{0} & \cdots & \mathbf{0} & H_{l_{1}-1} & H_{l_{1}-2} & \cdots & H_{0}
\end{array}\right]}_{l_{1}+k_{1}-1 \text { blocks }} \\
& \mathbf{s}=\left[s_{0,0}, s_{0,1}, \ldots, s_{0, n_{2}-1}, s_{1,0}, \ldots, s_{n_{1}-1, n_{2}-1}\right]^{T} \\
& S_{p_{1}}=\underbrace{\left[\begin{array}{cccc}
s_{p_{1}, 0} & s_{p_{1}, 1} & \cdots & s_{p_{1}, n_{2}-l_{2}-k_{2}+1} \\
s_{p_{1}, 1} & s_{p_{1}, 2} & \cdots & s_{p_{1}, n_{2}-l_{2}-k_{2}+2} \\
\vdots & \vdots & \vdots & \vdots \\
s_{p_{1}, l_{2}+k_{2}-2} & s_{p_{1}, l_{2}+k_{2}-1} & \cdots & s_{p_{1}, n_{2}-1}
\end{array}\right]}_{n_{2}-l_{2}-k_{2}+2 \text { blocks }} \\
& \mathbf{S}=\underbrace{\left[\begin{array}{cccc}
S_{0} & S_{1} & \cdots & S_{n_{1}-l_{1}-k_{1}+1} \\
S_{1} & S_{2} & \cdots & S_{n_{1}-l_{1}-k_{1}+2} \\
\vdots & \vdots & \vdots & \vdots \\
S_{l_{1}+k_{1}-2} & S_{l_{1}+k_{1}-1} & \cdots & S_{n_{1}-1}
\end{array}\right]}_{n_{1}-l_{1}-k_{1}+2 \text { blocks }}
\end{aligned}
$$


If the dimension of the null space of $\mathbf{V}$ is equal to one, then the original image can be exactly restored. For convenience, exact restoration means up to a scalar ambiguity. Throughout our discussion, we assume

A1) $l_{1}$ and $l_{2}$ are known;

A2) $\quad \mathbf{H}$ has full column rank;

A3) dimension of the null space of $\mathbf{V}$ is equal to one.

Several approaches can be found in [21] and [22] to determine $l_{1}$ and $l_{2}$. The other assumptions can be satisfied under some mild conditions [28].

\section{B. Formulation of Optimization Problems}

In noisy cases, $\mathcal{N}\{\mathbf{X}\}$ is an estimate of $\mathcal{N}\{\mathbf{S}\}$ and, usually, the matrix $\mathcal{N}\{\mathbf{X}\}$ is an $\left(\left(n_{1}-r_{1}\right)\left(n_{2}-r_{2}\right)-m k_{1} k_{2}\right) \times\left(n_{1}-\right.$ $\left.r_{1}\right)\left(n_{2}-r_{2}\right)$ matrix. $\mathcal{R}\left\{\mathbf{V}^{\prime}\right\} \neq \mathcal{R}\{\mathbf{V}\}$. Exact restoration is impossible. We can estimate the original image $s$ by solving the following optimization problem

$$
\begin{aligned}
\arg \min _{y}\|\mathbf{Y N}\{\mathbf{X}\}\|^{2} & \\
& \text { subject to }\|\mathbf{y}\|^{2}=1
\end{aligned}
$$

where $\mathbf{Y}$ and $\mathbf{y}$ are corresponding matrices of $y$ as $s$ is to $\mathbf{S}$ and $\mathbf{s}$, respectively. The solution of (3) is equal to the right singular vector corresponding to the smallest singular value of $\mathbf{V}$. Alternatively, the original image can also be estimated by solving the following optimization problem:

$$
\begin{aligned}
& \underset{y}{\arg \min _{y}}\|\mathbf{Y N}\{\mathbf{X}\}\|^{2} \\
& \text { subject to }\left\|\mathbf{y}_{p_{1}, p_{2}}\right\|^{2}=\left\|\mathbf{s}_{p_{1}, p_{2}}\right\|^{2} \\
& \text { for } p_{1}=0,1, \ldots, r_{1} \text { and } p_{2}=0,1, \ldots, r_{2}
\end{aligned}
$$

where $\mathbf{s}_{p_{1}, p_{2}}^{T}$ and $\mathbf{y}_{p_{1}, p_{2}}^{T}$ are the $\left(p_{1} r_{2}+p_{2}\right)$ th row of $\mathbf{S}$ and $\mathbf{Y}$, respectively. The difference between (3) and (4) is the constraint. Obviously, in the noise-free case, these two problems have the original image as their solution. Actually, we can find the solution of (3) by solving (2). The Lagrangian of (4) is

$$
\|\mathbf{Y N}\{\mathbf{X}\}\|^{2}+\sum_{p_{1}=0}^{r_{1}} \sum_{p_{2}=0}^{r_{2}} \lambda_{p_{1}, p_{2}}\left(\left\|\mathbf{y}_{p_{1}, p_{2}}\right\|^{2}-\left\|\mathbf{s}_{p_{1}, p_{2}}\right\|^{2}\right)
$$

where $\lambda_{p_{1}, p_{2}}$ are called Lagrange multipliers. However, it is impossible to find the optimal solution of the problem because the $\left\|\mathbf{s}_{p_{1}, p_{2}}\right\|^{2}$ terms are unknown. To release the constraint in (4) by setting $\lambda_{p_{1}, p_{2}}=\lambda$, (5) becomes

$$
\|\mathbf{Y N}\{\mathbf{X}\}\|^{2}+\lambda\left(\|\mathbf{Y}\|^{2}-\|\mathbf{S}\|^{2}\right) .
$$

Therefore, we obtain another constrained optimization problem

$$
\begin{aligned}
\arg \min _{y}\|\mathbf{Y N}\{\mathbf{X}\}\|^{2} & \text { subject to }\|\mathbf{Y}\|^{2}=1 .
\end{aligned}
$$

Lemma 1: Equation (7) has $\mathbf{s}$ as the unique solution in the noise-free case.

Proof: See Appendix A-1.

Thus, the original image can also be restored by solving (7).

On the other hand, if the matrix $\mathbf{H}$ has full column rank, then the column space $\mathcal{R}\left\{\mathbf{X}^{\mathcal{T}}\right\}$ of $\mathbf{X}^{T}$ is equal to the column space $\mathcal{R}\left\{\mathbf{S}^{\mathcal{T}}\right\}$ of $\mathbf{S}^{T}$ in the noise-free case. In the noisy case, $\mathcal{R}\left\{\mathbf{X}^{\mathcal{T}}\right\}$ is an $\left(n_{1}-r_{1}\right)\left(n_{2}-r_{2}\right) \times\left(r_{1}+1\right)\left(r_{2}+1\right)$ matrix and an estimate of $\mathcal{R}\left\{\mathbf{S}^{\mathcal{T}}\right\}$. Following the same argument as above, we form the optimization problem

$$
\begin{aligned}
\arg \max _{y}\left\|\mathbf{Y} \mathcal{R}\left\{\mathbf{X}^{T}\right\}\right\|^{2} \\
\\
\text { subject to }\|\mathbf{Y}\|^{2}=1 .
\end{aligned}
$$

$$
\begin{aligned}
\mathbf{v}_{p_{1}, p_{2}}= & \left(n_{2}-r_{2}\right) p_{1}+p_{2}, 0, v_{\left(n_{2}-r_{2}\right) p_{1}+p_{2}, 1}, \ldots, \\
V_{p_{1}}= & \underbrace{\left.v_{\left(n_{2}-r_{2}\right) p_{1}+p_{2},\left(n_{1}-r_{1}\right)\left(n_{2}-r_{2}\right)-\left(r_{1}+1\right)\left(r_{2}+1\right)-1}\right]^{T}} \\
\mathbf{V}= & \underbrace{\left[\begin{array}{ccccccc}
V_{0} & V_{1} & \cdots & V_{n_{1}-r_{1}-1} & \mathbf{0} & \cdots & \mathbf{0} \\
\mathbf{0} & V_{0} & V_{1} & \cdots & V_{n_{1}-r_{1}-1} & \ddots & \mathbf{0} \\
\vdots & \ddots & \ddots & \ddots & \ddots & \ddots & \vdots \\
\mathbf{0} & \cdots & \mathbf{0} & V_{0} & V_{1} & \cdots & V_{n_{1}-r_{1}-1}
\end{array}\right]}_{\left.\begin{array}{ccccccc}
\mathbf{v}_{p_{1}, 0} & \mathbf{v}_{p_{1}, 1} & \cdots & \mathbf{v}_{p_{1}, n_{2}-r_{2}-1} & \mathbf{0} & \cdots & \mathbf{0} \\
\mathbf{0} & \mathbf{v}_{p_{1}, 0} & \mathbf{v}_{p_{1}, 1} & \cdots & \mathbf{v}_{p_{1}, n_{2}-r_{2}-1} & \ddots & \mathbf{0} \\
\vdots & \ddots & \ddots & \ddots & \ddots & \ddots & \vdots \\
\mathbf{0} & \cdots & \mathbf{0} & \mathbf{v}_{p_{1}, 0} & \mathbf{v}_{p_{1}, 1} & \cdots & \mathbf{v}_{p_{1}, n_{2}-r_{2}-1}
\end{array}\right]}
\end{aligned}
$$




\section{Matrix Operations}

Now, we would like to solve the optimization problems (7) and (8). Define

$$
\begin{aligned}
w_{p_{1}, p_{2}}= & \omega_{p_{1}} \omega_{p_{2}} \\
\mathbf{W}_{p_{1}}= & {\left[\begin{array}{cccc}
w_{p_{1}, 0} & 0 & \cdots & 0 \\
0 & w_{p_{1}, 1} & \ddots & 0 \\
\vdots & \ddots & \ddots & \ddots \\
0 & \cdots & 0 & w_{p_{1}, n_{2}-1}
\end{array}\right] } \\
\mathbf{W} & =\left[\begin{array}{cccc}
\mathbf{W}_{0} & 0 & \cdots & 0 \\
0 & \mathbf{W}_{1} & \ddots & 0 \\
\vdots & \ddots & \ddots & \ddots \\
0 & \cdots & 0 & \mathbf{W}_{n_{1}-1}
\end{array}\right]
\end{aligned}
$$

where

$$
\begin{gathered}
\omega_{p 1}=\left\{\begin{array}{cc}
\frac{1}{\sqrt{p_{1}+1}} & 0 \leq p_{1} \leq r_{1}-1 \\
\frac{1}{\sqrt{r_{1}+1}} & r_{1} \leq p_{1} \leq n_{1}-r_{1}-1 \\
\frac{1}{\sqrt{n_{1}-p_{1}}} & n_{1}-r_{1} \leq p_{1} \leq n_{1}-1
\end{array}\right. \\
\omega_{p 2}=\left\{\begin{array}{cc}
\frac{1}{\sqrt{p_{2}+1}} & 0 \leq p_{2} \leq r_{2}-1 \\
\frac{1}{\sqrt{r_{2}+1}} & r_{2} \leq p_{2} \leq n_{2}-r_{2}-1 \\
\frac{1}{\sqrt{n_{2}-p_{2}}} & n_{2}-r_{2} \leq p_{2} \leq n_{2}-1
\end{array} .\right.
\end{gathered}
$$

Notice that $b W$ is an $n_{1} n_{2} \times n_{1} n_{2}$ matrix. Let $\tilde{\mathbf{V}}=\mathbf{V W}$. The next lemma implies a method to obtain the unique solution of (7) using matrix operations.

Lemma 2: The right singular vector corresponding to the smallest singular value of $\tilde{\mathbf{V}}$ is equal to the product of the matrix $\mathbf{W}^{-1}$ and the unique solution of (7).

Proof: See Appendix A-2.

In other words, the product of the matrix $\mathbf{W}$ and the right singular vector corresponding to the smallest singular value of $\tilde{\mathbf{V}}$ is equal to the unique solution of (7).

Lemma 3: Equation (8) has $\mathbf{s}$ as the unique solution in the noise-free case.
Proof: See Appendix A-3.

Let $u_{q_{1}, q_{2}}$ be the $\left(q_{1}, q_{2}\right)$ entry of $\mathcal{R}\left\{\mathbf{X}^{T}\right\}$ and (see the equation shown at the bottom of the page). Notice that $\mathbf{u}_{p_{1}, p_{2}}, \mathbf{U}_{p_{1}}$ and $\mathbf{U}$ are defined similar to $\mathbf{v}_{p_{1}, p_{2}}, \mathbf{V}_{p_{1}}$ and $\mathbf{V}$. The matrix $\mathbf{U}$ is an $\left(r_{1}+1\right)^{2}\left(r_{2}+1\right)^{2} \times n_{1} n_{2}$ matrix. Let $\tilde{\mathbf{U}}=\mathbf{U W}$.

Lemma 4: The right singular vector corresponding to the largest singular value of $\tilde{\mathbf{U}}$ is equal to the product of the matrix $\mathbf{W}^{-1}$ and the unique solution of (8).

Proof: See Appendix A-4.

In other words, the product of the matrix $\mathrm{W}$ and the right singular vector corresponding to the largest singular value of $\tilde{\mathbf{U}}$ is equal to the unique solution of (8).

\section{IMPLEMENTATION}

\section{A. Two Algorithms}

Based on the derivation in the section above, two algorithms for direct multichannel blind image restoration are summarized as follows. The first algorithm exploits the null space of the matrix $\mathbf{X}$ of the blurred images.

1) Construct $X$ from the blurred images.

2) Estimate the null space $\mathcal{N}\{\mathbf{S}\}$ of $\mathbf{S}$ from the null space $\mathcal{N}\{\mathbf{X}\}$ of $\mathbf{X}$.

3) Construct the matrix $\mathbf{V}$ from the orthonormal basis $\mathbf{N}_{\mathbf{S}}$ of estimated $\mathcal{N}\{\mathbf{S}\}$.

4) Obtain the matrix $\mathbf{V}$ by multiplying $\mathbf{V}$ with the diagonal matrix $\mathbf{W}$.

5) Find the product of $\mathbf{W}$ and the right singular vector corresponding to the smallest singular value of $\tilde{\mathbf{V}}$.

The product is the restored image. The second algorithm is based on the column space of $\mathbf{X}$.

1) Construct $X$ from the blurred images.

2) Estimate the column space $\mathcal{R}\left\{\mathbf{S}^{\mathcal{T}}\right\}$ of $\mathbf{S}$ from the column space $\mathcal{R}\left\{\mathbf{X}^{\mathcal{T}}\right\}$ of $\mathbf{X}$.

3) Construct the matrix $\mathbf{U}$ from the orthonormal basis $\mathbf{R}_{\mathbf{S}^{T}}$ of estimated $\mathcal{R}\left\{\mathbf{S}^{\mathcal{T}}\right\}$

4) Obtain the matrix $\tilde{\mathbf{U}}$ by multiplying $\mathbf{U}$ with the diagonal matrix $\mathbf{W}$.

$$
\begin{aligned}
& \mathbf{u}_{p_{1}, p_{2}}=\left[u_{\left(n_{2}-r_{2}\right) p_{1}+p_{2}, 0}, u_{\left(n_{2}-r_{2}\right) p_{1}+p_{2}, 1}, \ldots, u_{\left(n_{2}-r_{2}\right) p_{1}+p_{2},\left(r_{1}+1\right)\left(r_{2}+1\right)-1}\right]^{T} \\
& \mathbf{U}_{p_{1}}=\underbrace{\left[\begin{array}{ccccccc}
\mathbf{u}_{p_{1}, 0} & \mathbf{u}_{p_{1}, 1} & \cdots & \mathbf{u}_{p_{1}, n_{2}-r_{2}-1} & \mathbf{0} & \cdots & \mathbf{0} \\
\mathbf{0} & \mathbf{u}_{p_{1}, 0} & \mathbf{u}_{p_{1}, 1} & \cdots & \mathbf{u}_{p_{1}, n_{2}-r_{2}-1} & \ddots & \mathbf{0} \\
\ddots & \ddots & \ddots & \ddots & \ddots & \ddots & \vdots \\
\mathbf{0} & \cdots & \mathbf{0} & \mathbf{u}_{p_{1}, 0} & \mathbf{u}_{p_{1}, 1} & \cdots & \mathbf{u}_{p_{1}, n_{2}-r_{2}-1}
\end{array}\right]}_{n_{2} \text { blocks }} \\
& \mathbf{U}=\underbrace{\left[\begin{array}{ccccccc}
\mathbf{U}_{0} & \mathbf{U}_{1} & \cdots & \mathbf{U}_{n_{1}-r_{1}-1} & \mathbf{0} & \cdots & \mathbf{0} \\
\mathbf{0} & \mathbf{U}_{0} & \mathbf{U}_{1} & \cdots & \mathbf{U}_{n_{1}-r_{1}-1} & \ddots & \mathbf{0} \\
\ddots & \ddots & \ddots & \ddots & \ddots & \ddots & \vdots \\
\mathbf{0} & \cdots & \mathbf{0} & \mathbf{U}_{0} & \mathbf{U}_{1} & \cdots & \mathbf{U}_{n_{1}-r_{1}-1}
\end{array}\right]}_{n_{1} \text { blocks }} \\
& n_{1} \text { blocks }
\end{aligned}
$$


5) Find the product of $\mathbf{W}$ and the right singular vector corresponding to the largest singular value of $\tilde{\mathbf{U}}$.

Again, the product is the restored image.

In the noise-free case, both algorithms exactly restore the original image, up to a scalar ambiguity. In practice, the noise terms $e^{(i)}$ in (1) cannot be ignored. Define

$$
\begin{aligned}
& \mathbf{e}_{p_{1}, p_{2}}= {\left[e_{p_{1}, p_{2}}^{(1)}, e_{p_{1}, p_{2}}^{(2)}, \ldots, e_{p_{1}, p_{2}}^{(m)}\right]^{T} } \\
& E_{p_{1}}= {\left[\begin{array}{cccc}
\mathbf{e}_{p_{1}, l_{2}-1} & \mathbf{e}_{p_{1}, l_{2}} & \cdots & \mathbf{e}_{p_{1}, n_{2}-k_{2}} \\
\mathbf{e}_{p_{1}, l_{2}} & \mathbf{e}_{p_{1}, l_{2}+1} & \cdots & \mathbf{e}_{p_{1}, n_{2}-k_{2}+1} \\
\vdots & \vdots & \vdots & \vdots \\
\mathbf{e}_{p_{1}, l_{2}+k_{2}-2} & \mathbf{e}_{p_{1}, l_{2}+k_{2}-1} & \cdots & \mathbf{e}_{p_{1}, n_{2}-1}
\end{array}\right] } \\
& \mathbf{E}=\left[\begin{array}{cccc}
E_{l_{1}-1} & E_{l_{1}} & \cdots & E_{n_{1}-k_{1}} \\
E_{l_{1}} & E_{l_{1}+1} & \cdots & E_{n_{1}-k_{1}+1} \\
\vdots & \vdots & \vdots & \vdots \\
E_{l_{1}+k_{1}-2} & E_{l_{1}+k_{1}-1} & \cdots & E_{n_{1}-1}
\end{array}\right] .
\end{aligned}
$$

Therefore

$$
\mathbf{X}=\mathbf{H S}+\mathbf{E} .
$$

Generally, because $\mathcal{N}\{\mathbf{X}\} \neq \mathcal{N}\{\mathbf{S}\}$ and $\mathcal{R}\left\{\mathbf{X}^{\mathcal{T}}\right\} \neq \mathcal{R}\left\{\mathbf{S}^{\mathcal{T}}\right\}$ when there is noise, exact restoration of the original image is impossible. The bases $\mathbf{N}_{\mathbf{S}}$ and $\mathbf{R}_{\mathbf{S}^{T}}$ are estimated from $\mathbf{X}$ and $\left(\mathbf{N}_{\mathbf{S}}\right)^{T} \mathbf{R}_{\mathbf{S}^{T}}=\mathbf{0}$. The results of both algorithms are the leastsquares estimates of the original image. In fact, the results are the same as shown in the following lemma.

Lemma 5: The right singular vector corresponding to the smallest singular value of $\tilde{\mathbf{V}}$ is equal to the right singular vector corresponding to the largest singular value of $\tilde{\mathbf{U}}$.

Proof: From the definition of $\mathbf{R}_{\mathbf{S}^{T}}$ and $\mathbf{N}_{\mathbf{S}}$, $\left[\mathbf{R}_{\mathbf{S}^{T}} \mathbf{N}_{\mathbf{S}}\right]$ is a unitary matrix. That is

$$
\left[\mathbf{R}_{\mathbf{S}^{T}} \mathbf{N}_{\mathbf{S}}\right]\left[\begin{array}{c}
\left(\mathbf{R}_{\mathbf{S}^{T}}\right)^{T} \\
\left(\mathbf{N}_{\mathbf{S}}\right)^{T}
\end{array}\right]=\mathbf{I} .
$$

As a result

$$
\mathbf{R}_{\mathbf{S}^{T}}\left(\mathbf{R}_{\mathbf{S}^{T}}\right)^{T}+\mathbf{N}_{\mathbf{S}}\left(\mathbf{N}_{\mathbf{S}}\right)^{T}=\mathbf{I}
$$

Recalling that $\mathbf{U}$ and $\mathbf{V}$ are constructed from $\mathbf{R}_{\mathbf{S}^{T}}$ and $\mathbf{N}_{\mathbf{S}}$ respectively, we obtain

$$
\mathbf{U}^{T} \mathbf{U}+\mathbf{V}^{T} \mathbf{V}=\left(\mathbf{W}^{-1}\right)^{2}
$$

based on (9). Therefore

$$
\begin{aligned}
& \mathbf{W}^{T} \mathbf{U}^{T} \mathbf{U} \mathbf{W}+\mathbf{W}^{T} \mathbf{V}^{T} \mathbf{V W} \\
= & \mathbf{W}^{T}\left(\mathbf{W}^{-1}\right)^{2} \mathbf{W} \\
\Longrightarrow & \tilde{\mathbf{U}}^{T} \tilde{\mathbf{U}}+\tilde{\mathbf{V}}^{T} \tilde{\mathbf{V}}=\mathbf{I} .
\end{aligned}
$$

Assume the eigenvalue decomposition of $\tilde{\mathbf{U}}^{T} \tilde{\mathbf{U}}$ equals $U^{T} D U$, where $U$ is a unitary matrix and $D$ is a diagonal matrix. Because $\tilde{\mathbf{U}}^{T} \tilde{\mathbf{U}}$ is positive semidefinite, all of its eigenvalues are nonnegative [29]. Then

$$
\begin{aligned}
& D+U \tilde{\mathbf{V}}^{T} \tilde{\mathbf{V}} U^{T}=\mathbf{I} \\
\Longrightarrow & U \tilde{\mathbf{V}}^{T} \tilde{\mathbf{V}} U^{T}=\mathbf{I}-D \\
\Longrightarrow & \tilde{\mathbf{V}}^{T} \tilde{\mathbf{V}}=U^{T}(\mathbf{I}-D) U .
\end{aligned}
$$

Since $U$ is a unitary matrix and $\mathbf{I}-D$ is a diagonal matrix, the eigenvalue decomposition of $\tilde{\mathbf{V}}^{T} \tilde{\mathbf{V}}$ equals $U^{T}(\mathbf{I}-D) U$. Thus, the eigenvector corresponding to the smallest eigenvalue of $\tilde{\mathbf{U}}^{T} \tilde{\mathbf{U}}$ equals the eigenvector corresponding to the largest eigenvalue of $\tilde{\mathbf{V}}^{T} \tilde{\mathbf{V}}$.

Remarks 1: Since $\tilde{\mathbf{V}}^{T} \tilde{\mathbf{V}}$ is also positive semidefinite, all of its eigenvalues are nonnegative. Therefore, all eigenvalues of $\tilde{\mathbf{U}}^{T} \tilde{\mathbf{U}}$ and $\tilde{\mathbf{V}}^{T} \tilde{\mathbf{V}}$ are less than or equal to one. In fact, the smallest eigenvalue of $\tilde{\mathbf{V}}^{T} \tilde{\mathbf{V}}$ and the largest eigenvalue of $\tilde{\mathbf{U}}^{T} \tilde{\mathbf{U}}$ are zero and one, respectively, in the noise-free case because of the unique solution of (7).

\section{B. Computational Complexity}

Define

$$
\begin{aligned}
n & =n_{1} n_{2} \\
n_{r} & =\left(n_{1}-r_{1}\right)\left(n_{2}-r_{2}\right) \\
r & =\left(r_{1}+1\right)\left(r_{2}+1\right) \\
k & =k_{1} k_{2} .
\end{aligned}
$$

We use LAPACK [30] to do matrix operations. The bases $\mathbf{N}_{\mathbf{S}}$ and $\mathbf{R}_{\mathbf{S}^{T}}$ are $n_{r} \times n_{r}-r$ and $n_{r} \times r$ matrices respectively. We assume that there is noise throughout the rest of this section.

The $Q R$ decomposition [31] is used to estimate $\mathbf{N}_{\mathbf{S}}$ and $\mathbf{R}_{\mathbf{S}^{T}}$. Table I shows the computational complexities of these two algorithms using the QR decomposition in Step 2. Notice that one flop and one word are, respectively, equal to one floating point operation and the number of bytes required for a floating point number. When $n_{r}-r>r$, the computational complexity of the second algorithm is lower than the first one.

The matrices $\mathbf{V}$ and $\mathbf{U}$ constructed in Step 3 of these two algorithms are $\left(n_{r}-r\right) r \times n$ and $r^{2} \times n$, respectively. The storage requirements for explicitly storing these two matrices are huge. In addition, the computational costs of Step 5 of both algorithms are unacceptable because of the required operations on the huge matrices.

Rather, we use the power method [31] to implement Steps 4 and 5. This method computes the eigenvector corresponding to the largest eigenvalue of $\tilde{\mathbf{U}}^{T} \tilde{\mathbf{U}}$ without explicitly constructing the matrices $\tilde{\mathbf{U}}$ and $\tilde{\mathbf{U}}^{T} \tilde{\mathbf{U}}$. The eigenvector actually is the right singular vector corresponding to the largest singular value of $\tilde{\mathbf{U}}$.

From Remark I, the largest eigenvalue of $\tilde{\mathbf{V}}^{T} \tilde{\mathbf{V}}$ is less than or equal to one. As a result, this method computes the eigenvector corresponding to the largest eigenvalue of $\tilde{\mathbf{V}}^{T} \tilde{\mathbf{V}}-\mathbf{I}$ without explicitly constructing the matrices $\tilde{\mathbf{V}}$ and $\tilde{\mathbf{V}}^{T} \tilde{\mathbf{V}}-\mathbf{I}$. The eigenvector actually is the right singular vector corresponding to the smallest singular value of $\tilde{\mathbf{V}}$. Table II shows the computational complexities of these two algorithms in Steps 4 and 5. Again, when $n_{r}-r>r$, the computational complexity of the second algorithm is lower than the first one. Notice that the computational cost in Table II is estimated based on each iteration of the power method. The number of iterations is dependent on the ratio between the largest and the second largest singular values of the matrices $\tilde{\mathbf{U}}^{T} \tilde{\mathbf{U}}^{\text {and }} \tilde{\mathbf{V}}^{T} \tilde{\mathbf{V}}-\mathbf{I}$. In simulations, the number of iterations is typically around 70 .

From Tables I and II, because in practice $n_{r}$ is greater than $2 r$, the computational complexity of the first algorithm is greater than the second one. When we have more blurred versions of 
TABLE I

COMPUTATIONAL COMPLEXITIES OF STEP 2 IN THE FIRST AND SECOND ALGORITHMS USING THE QR DECOMPOSITION

\begin{tabular}{l|c|c}
\hline & First Algorithm & Second Algorithm \\
\hline Computational Cost (flops) & $\begin{array}{l}4 n_{r}\left(n_{r}-r\right)^{2} \\
-\frac{4}{3}\left(\left(n_{r}-r\right)^{3}-r^{3}\right) \\
+3 r^{2}\left(n_{r}-r\right)\end{array}$ & $4 n_{r} r^{2}-\frac{4}{3} r^{3}$ \\
\hline Storage Requirement (words) & $n_{r}\left(n_{r}-r\right)+r^{2}$ & $n_{r} r$ \\
\hline
\end{tabular}

TABLE II

COMPUTATIONAL COMPLeXITIES OF STEPS 4 AND 5 In the FiRST AND SeCOND ALGORITHMS USING THE POWER METHOD

\begin{tabular}{l|c|c}
\hline & First Algorithm & Second Algorithm \\
\hline Computational Cost (flops) & $2 n_{r}\left(n_{r}-r\right)^{2}$ & $2 n_{r} r^{2}$ \\
\hline Storage Requirement (words) & $\left(n_{r}+r\right)\left(n_{r}-r\right)+n$ & $n_{r} r+n+r^{2}$ \\
\hline
\end{tabular}

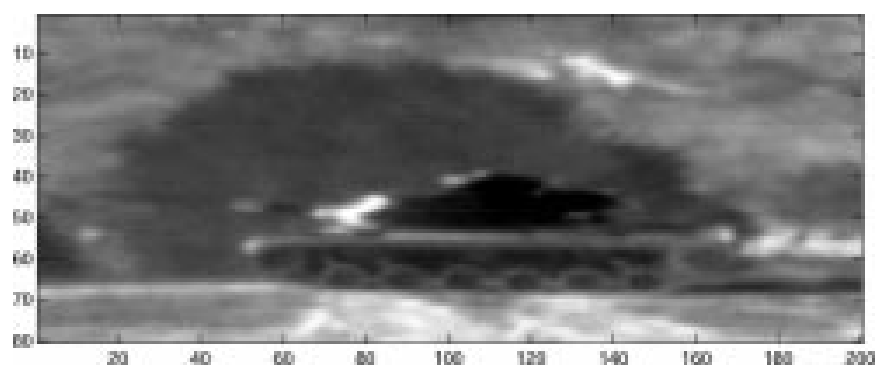

Fig. 2. Original infrared images of an M60 tank: $80 \times 200$ tank image.

the original image, $k_{1}$ and $k_{2}$ can be smaller, thus lowering the complexity. If $r^{2} \approx m \log _{2} n$, the computational complexity of the second algorithm is close to the FFT complexity, $m n \log _{2} n$. Furthermore, Lemma 5 shows that the results of both algorithms are the same. Thus, we will use the second algorithm for discussion in the following sections.

\section{CONNECTIONS TO THE RESTORATION FILTER Estimation AlgORITHM}

The connection between the least-squares and the subspace approaches to blind channel estimation was established in [32]. In [25], the relation between the direct least-squares approach [19] to the blind multichannel equalizer identification and the subspace intersection method to the blind multichannel signal estimation [24] was studied. In this section, we compare the second algorithm in the last section with the algorithm proposed by Giannakis et al. based on the idea in [25].

Let

$$
\mathrm{X}^{T}=Q R \quad: \mathrm{QR} \text { decomposition }
$$

where Q: $\left(n_{1}-r_{1}\right)\left(n_{2}-r_{2}\right) \times m k_{1} k_{2}$ matrix with $m k_{1} k_{2}$ orthonormal columns and $R: m k_{1} k_{2} \times m k_{1} k_{2}$ upper triangular matrix $Q$ is a base of $\mathcal{R}\left\{\mathbf{X}^{\mathcal{T}}\right\}$, that is, a basis estimate of $\mathcal{R}\left\{\mathbf{S}^{\mathcal{T}}\right\}$.

In Step 5 of the second algorithm, the right singular vector $\tilde{\mathbf{y}}_{\text {opt }}$ corresponding to the largest singular value of $\tilde{\mathbf{U}}$ can be written as follows:

$$
\tilde{\mathbf{y}}_{\text {opt }}=\arg \max _{\|\tilde{\mathbf{y}}\|^{2}=1}\|\tilde{\mathbf{U}} \tilde{\mathbf{y}}\|^{2} .
$$

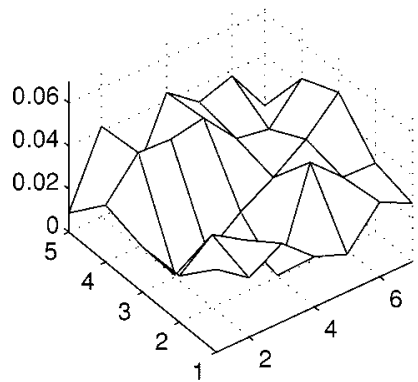

(a)

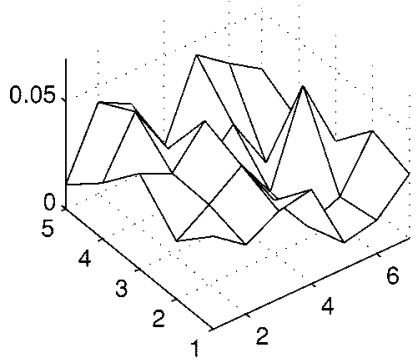

(c)

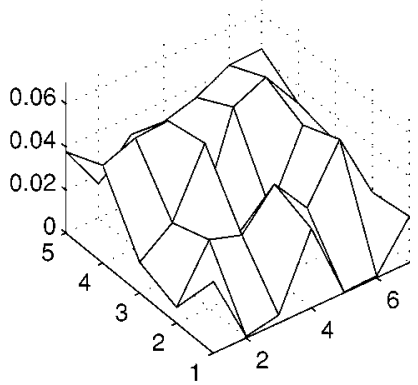

(b)

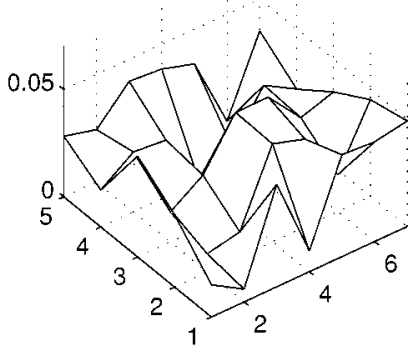

(d)
Fig. 3. Four $5 \times 7$ blur functions.

Define an $m k_{1} k_{2}\left(r_{1}+1\right)\left(r_{2}+1\right) \times 1$ vector $\tilde{\mathbf{z}}_{\text {opt }}$ to be the left singular vector corresponding to the largest singular value of $\tilde{\mathbf{U}}$. Therefore, $\tilde{\mathbf{z}}_{\text {opt }}^{T} \tilde{\mathbf{U}}=\tilde{\mathbf{y}}_{\text {opt }}$ In other words

$$
\begin{aligned}
\tilde{\mathbf{z}}_{\text {opt }} & =\arg \max _{\|\tilde{\mathbf{z}}\|^{2}=1}\left\|\tilde{\mathbf{z}}^{T} \tilde{\mathbf{U}}\right\|^{2} \\
& =\arg \max _{\tilde{\mathbf{z}}^{T} \tilde{\mathbf{Z}}=1} \tilde{\mathbf{z}}^{T} \tilde{\mathbf{U}} \tilde{\mathbf{U}}^{T} \tilde{\mathbf{z}} .
\end{aligned}
$$

Let $Q$ be the estimated basis $\mathbf{R}_{\mathbf{S}^{T}}$ of $\mathcal{R}\left\{\mathbf{S}^{\mathcal{T}}\right\}$. Define (see the first equation shown at the bottom of the next page) where $\mathbf{X}_{p_{1}}$ is defined as in Section III-A. Thus

$$
\tilde{z}_{\mathrm{opt}}=\arg \max _{\tilde{z}^{T} \tilde{z}=1} \tilde{z}^{T} \mathbf{R} \chi \mathbf{W} \mathbf{W}^{T} \chi^{T} \mathbf{R}^{T} \tilde{z} .
$$

Let $\tilde{\mathbf{g}}=\mathbf{R}^{T} \tilde{z}$. Because

$$
\begin{aligned}
R^{T} R & =R^{T} Q^{T} Q R \\
& =\mathbf{X X}^{T}
\end{aligned}
$$

we obtain

$$
\begin{aligned}
& \tilde{\mathbf{z}}^{T} \tilde{\mathbf{z}}=1 \\
\Longrightarrow & \tilde{\mathbf{g}}^{T} \mathbf{R}^{-1}\left(\mathbf{R}^{-1}\right)^{T} \tilde{\mathbf{g}}=1 \\
\Longrightarrow & \tilde{\mathbf{g}}^{T} C_{\mathbf{X}} \tilde{\mathbf{g}}=1
\end{aligned}
$$

where

$$
C_{\mathbf{X}}=\underbrace{\left[\begin{array}{cccc}
\mathbf{X X}^{T} & 0 & \cdots & 0 \\
0 & \mathbf{X X}^{T} & \ddots & 0 \\
\vdots & \ddots & \ddots & \ddots \\
0 & \cdots & 0 & \mathbf{X X}^{T}
\end{array}\right]}_{\left(r_{1}+1\right)\left(r_{2}+1\right) \text { blocks }}
$$


Therefore, from (11)

$$
\tilde{\mathbf{g}}_{\text {opt }}=\arg \max _{\tilde{\mathbf{g}}^{T} C_{\mathbf{X}} \tilde{\mathbf{g}}=1} \tilde{\mathbf{g}}^{T} \chi \mathbf{W} \mathbf{W}^{T} \chi^{T} \tilde{\mathbf{g}}
$$

That is, $\tilde{\mathbf{y}}_{\text {opt }}$ in (10) is equal to $\tilde{\mathbf{g}}_{\text {opt }}^{T} \chi \mathbf{W}$. The restored image obtained from the second algorithm is equal to $\tilde{\mathbf{g}}_{\text {opt }}^{T} \chi \mathbf{W}^{2}$. As a result, $\tilde{\mathbf{g}}_{\text {opt }}$ can be regarded as a vector of the restoration filters.

In [22], from (1), Giannakis et al. seek to find restoration filters $g^{\left(i, q_{1}, q_{2}\right)}$, such that

$$
\begin{aligned}
\sum_{i} x^{(i)} * g^{\left(i, q_{1}, q_{2}\right)} & =\sum_{i} s * h^{(i)} * g^{\left(i, q_{1}, q_{2}\right)} \\
& =s * \sum_{i} h^{(i)} * g^{\left(i, q_{1}, q_{2}\right)} \\
& =s^{\left(q_{1}, q_{2}\right)}
\end{aligned}
$$

where $s^{\left(q_{1}, q_{2}\right)}$ is a shifted version of $s$, that is $s_{p_{1}, p_{2}}^{\left(q_{1}, q_{2}\right)}=$ $s_{p_{1}-q_{1}, p_{2}-q_{2}}$. As a result

$$
\begin{aligned}
s_{p_{1}, p_{2}}^{\left(q_{1}, q_{2}\right)} & =\sum_{i} \sum_{j_{1}=0}^{\tilde{l}_{1}-1} \sum_{j_{2}=0}^{\tilde{l}_{2}-1} x_{p_{1}-j_{1}, p_{2}-j_{2}}^{(i)} g_{j_{1}, j_{2}}^{\left(i, q_{1}, q_{2}\right)} \\
& =s_{p_{1}-q_{1}, p_{2}-q_{2}} \\
& =\sum_{i} \sum_{j_{1}=0}^{\tilde{l}_{1}-1} \sum_{j_{2}=0}^{\tilde{l}_{2}-1} x_{p_{1}-q_{1}-j_{1}, p_{2}-q_{2}-j_{2}}^{(i)} g_{j_{1}, j_{2}}^{(i, 0,0)}
\end{aligned}
$$

Therefore, (see the second equation shown at the bottom of the page). Define $\tilde{l}_{1}=k_{1}, \tilde{l}_{2}=k_{2}$ and (see the equation shown at the bottom of the next page). Notice that the first zero block row in the definition of $\tilde{\mathbf{X}}_{p_{1}, p_{2}}$ has enough rows such that $\tilde{\mathbf{X}}_{p_{1}, p_{2}}$ has $m k_{1} k_{2}\left(r_{1}+1\right)\left(r_{2}+1\right)$ rows. In the noise-free case, we can find the unique solution for

$$
g^{T} \tilde{\mathbf{X}}=0
$$

$$
\begin{aligned}
& \chi_{p_{1}}=\underbrace{\left[\begin{array}{c}
X_{p_{1}} \\
X_{p_{1}+1} \\
\vdots \\
X_{p_{1}+k_{1}-1}
\end{array}\right]}_{n_{2}-r_{2} \text { columns }} \\
& \chi_{p_{1}}=\underbrace{\left[\begin{array}{cccc}
\chi_{p_{1}} & \mathbf{0} & \cdots & \mathbf{0} \\
\mathbf{0} & \chi_{p_{1}} & \ddots & \mathbf{0} \\
\vdots & \ddots & \ddots & \ddots \\
\mathbf{0} & \cdots & \mathbf{0} & \chi_{p_{1}}
\end{array}\right]}_{n_{2} \text { columns }} \\
& \chi=\underbrace{\left[\begin{array}{ccccccc}
\chi_{l_{1}-1} & \chi_{l_{1}} & \cdots & \chi_{n_{1}-k_{1}} & \mathbf{0} & \cdots & \mathbf{0} \\
\mathbf{0} & \chi_{l_{1}-1} & \chi_{l_{1}} & \cdots & \chi_{n_{1}-k_{1}} & \ddots & \mathbf{0} \\
\vdots & \ddots & \ddots & \ddots & \ddots & \ddots & \vdots \\
\mathbf{0} & \cdots & \mathbf{0} & \chi_{l_{1}-1} & \chi_{l_{1}} & \cdots & \chi_{n_{1}-k_{1}}
\end{array}\right]}_{n_{1} n_{2} \text { columns }} \\
& \mathbf{R}=\underbrace{\left[\begin{array}{cccc}
\left(\mathrm{R}_{\mathbf{X}^{T}}^{T}\right)^{-1} & \mathbf{0} & \cdots & \mathbf{0} \\
\mathbf{0} & \left(R_{\mathbf{X}^{T}}^{T}\right)^{-1} & \ddots & \mathbf{0} \\
\vdots & \ddots & \ddots & \ddots \\
\mathbf{0} & \cdots & \mathbf{0} & \left(R_{\mathbf{X}^{T}}^{T}\right)^{-1}
\end{array}\right]}_{\left(r_{1}+1\right)\left(r_{2}+1\right) \text { blocks }}
\end{aligned}
$$

$$
\sum_{i} \sum_{j_{1}=0}^{\tilde{l}_{1}-1} \sum_{j_{2}=0}^{\tilde{l}_{2}-1}\left(x_{p_{1}-q_{1}-j_{1}, p_{2}-q_{2}-j_{2}}^{(i)} g_{j_{1}, j_{2}}^{(i, 0,0)}-x_{p_{1}-j_{1}, p_{2}-j_{2}}^{(i)} g_{j_{1}, j_{2}}^{\left(i, q_{1}, q_{2}\right)}\right)=0
$$


That is, every pair of outputs of the restoration filters corresponding to the same pixel in the original image must have the same value. For example, the first column of $\tilde{\mathbf{X}}$ corresponds $s_{r_{1}, r_{2}}$. The product of $g^{T}$ and the first column is the difference between two restoration filters for $s_{r_{1}, r_{2}}$. The difference must be zero. Notice that there may be several columns of $\tilde{\mathbf{X}}$ corresponding to the same pixel of the original image.

In the noisy case, there is no solution for (15). All pairs of outputs are different. We would like to minimize the summation of the squares of all differences. The left singular vector $g_{1}$ corresponding to the smallest singular value of $\tilde{\mathbf{X}}$ could be an estimate of the vector $g$ of the restoration filters. That is

$$
\mathbf{g}_{1}=\arg \min _{\mathbf{g}^{T} \mathbf{g}=1} \mathbf{g}^{T} \tilde{\mathbf{X}} \tilde{\mathbf{X}}^{T} \mathbf{g}
$$

Here, we use a quadratic constraint on the restoration filters.

Define (see equation shown at the bottom of the next page) where $w_{p_{1}, p_{2}}$ are defined as in Section III-B. Let $\hat{\mathbf{X}}=\tilde{\mathbf{X}} \hat{\mathbf{W}}$. That is, we put different weights on different columns of $\tilde{\mathbf{X}}$. The columns corresponding to the same pixel have the same weight. If the number of columns corresponding to the same pixel is larger, the weight is smaller. Furthermore, rather than using the quadratic constraint on the restoration filters, we choose a quadratic constraint on the restored image, that is, $\mathbf{g}^{T} C_{\mathbf{X}} \mathbf{g}=1$. A vector $\hat{\mathrm{g}}_{\text {opt }}$ of different restoration filters can be found as follows:

$$
\hat{\mathbf{g}}_{\text {opt }}=\arg \min _{\mathbf{g}^{T}} C_{\mathbf{X}} \mathbf{g}=1 \mathbf{g}^{T} \hat{\mathbf{X}} \hat{\mathbf{X}}^{T} \mathbf{g}
$$

$$
\begin{aligned}
& \underline{\mathbf{X}}_{p_{1}}^{\left(q_{2}\right)}=\underbrace{\left[\begin{array}{cccc}
\mathbf{x}_{p_{1}, l_{2}-1} & \mathbf{x}_{p_{1}, l_{2}} & \cdots & \mathbf{x}_{p_{1}, n_{2}-k_{2}-q_{2}} \\
\mathbf{x}_{p_{1}, l_{2}} & \mathbf{x}_{p_{1}, l_{2}+1} & \cdots & \mathbf{x}_{p_{1}, n_{2}-k_{2}-q_{2}+1} \\
\vdots & \vdots & \vdots & \vdots \\
\mathbf{x}_{p_{1}, r_{2}} & \mathbf{x}_{p_{1}, r_{2}+1} & \cdots & \mathbf{x}_{p_{1}, n_{2}-q_{2}-1}
\end{array}\right]}_{n_{2}-r_{2}-q_{2} \text { blocks }} \\
& \underline{\mathbf{X}}^{\left(q_{1}, q_{2}\right)}=\underbrace{\left[\begin{array}{cccc}
\underline{\mathbf{X}}_{l_{1}-1}^{\left(q_{2}\right)} & \underline{\mathbf{X}}_{l_{1}}^{\left(q_{2}\right)} & \cdots & \underline{\mathbf{X}}_{n_{1}-k_{1}-q_{1}}^{\left(q_{2}\right)} \\
\underline{\mathbf{X}}_{l_{1}}^{\left(q_{2}\right)} & \underline{\mathbf{X}}_{l_{1}+1}^{\left(q_{2}\right)} & \cdots & \underline{\mathbf{X}}_{n_{1}-k_{1}-q_{1}+1}^{\left(q_{2}\right)} \\
\vdots & \vdots & \vdots & \vdots \\
\underline{\mathbf{X}}_{r_{1}}^{\left(q_{2}\right)} & \underline{\mathbf{x}}_{r_{1}+1}^{\left(q_{2}\right)} & \cdots & \underline{\mathbf{X}}_{n_{1}-q_{1}-1}^{\left(q_{2}\right)}
\end{array}\right]}_{n_{1}-r_{1}-q_{1} \text { blocks }} \\
& \overline{\mathbf{X}}_{p_{1}}^{\left(q_{2}\right)}=\underbrace{\left[\begin{array}{cccc}
\mathbf{x}_{p_{1}, l_{2}+q_{2}-1} & \mathbf{x}_{p_{1}, l_{2}+q_{2}} & \cdots & \mathbf{x}_{p_{1}, n_{2}-k_{2}} \\
\mathbf{x}_{p_{1}, l_{2}+q_{2}} & \mathbf{x}_{p_{1}, l_{2}+q_{2}+1} & \cdots & \mathbf{x}_{p_{1}, n_{2}-k_{2}+1} \\
\vdots & \vdots & \vdots & \vdots \\
\mathbf{x}_{p_{1}, r_{2}+q_{2}} & \mathbf{x}_{p_{1}, r_{2}+q_{2}+1} & \cdots & \mathbf{x}_{p_{1}, n_{2}-1}
\end{array}\right]}_{n_{2}-r_{2}-q_{2} \text { blocks }} \\
& \overline{\mathbf{X}}^{\left(q_{1}, q_{2}\right)}=\underbrace{\left[\begin{array}{cccc}
\overline{\mathbf{X}}_{l_{1}+q_{1}-1}^{\left(q_{2}\right)} & \overline{\mathbf{X}}_{l_{1}+q_{1}}^{\left(q_{2}\right)} & \cdots & \overline{\mathbf{X}}_{n_{1}-k_{1}}^{\left(q_{2}\right)} \\
\overline{\mathbf{X}}_{l_{1}+q_{1}}^{\left(q_{2}\right)} & \overline{\mathbf{X}}_{l_{1}+q_{1}+1}^{\left(q_{2}\right)} & \cdots & \mathbf{X}_{n_{1}-k_{1}+1}^{\left(q_{2}\right)} \\
\vdots & \vdots & \vdots & \vdots \\
\overline{\mathbf{X}}_{r_{1}+q_{1}}^{\left(q_{2}\right)} & \overline{\mathbf{X}}_{r_{2}+q_{1}+1}^{\left(q_{2}\right)} & \cdots & \overline{\mathbf{X}}_{n_{1}-1}^{\left(q_{2}\right)}
\end{array}\right]}_{n_{1}-r_{1}-q_{1} \text { blocks }} \\
& \tilde{\mathbf{X}}_{p_{1}, p_{2}}=\underbrace{\left[\begin{array}{cccccc}
0 & \cdots & \cdots & \cdots & \cdots & 0 \\
\underline{\mathbf{X}}^{(0,1)} & \cdots & \underline{\mathbf{X}}^{\left(0, r_{2}-p_{2}\right)} & \underline{\mathbf{X}}^{(1,0)} & \cdots & \underline{\mathbf{X}}^{\left(r_{1}-p_{1}, r_{2}-p_{2}\right)} \\
-\overline{\mathbf{X}}^{(0,1)} & 0 & \cdots & \cdots & \cdots & 0 \\
\vdots & \ddots & \ddots & \ddots & \ddots & \ddots \\
\vdots & \ddots & -\overline{\mathbf{X}}^{\left(0, r_{2}-p_{2}\right)} & \ddots & \ddots & \ddots \\
\vdots & \ddots & \ddots & -\overline{\mathbf{X}}^{(1,0)} & \ddots & \ddots \\
\vdots & \ddots & \ddots & \ddots & \ddots & \ddots \\
0 & \cdots & \cdots & \cdots & 0 & -\overline{\mathbf{X}}^{\left(r_{1}-p_{1}, r_{2}-p_{2}\right)}
\end{array}\right]} \\
& \left(r_{1}-p_{1}+1\right)\left(r_{2}-p_{2}+1\right)-1 \text { blocks } \\
& \tilde{\mathbf{X}}=\left[\tilde{\mathbf{X}}_{0,0}, \ldots, \tilde{\mathbf{X}}_{0, r_{2}}, \tilde{\mathbf{X}}_{1,0}, \ldots, \tilde{\mathbf{X}}_{r_{1}, r_{2}-1}\right]
\end{aligned}
$$


In the noise-free case, $\mathbf{g}_{1}^{T} \tilde{\mathbf{X}}=0$ from (15) and (16). Therefore, $\hat{\mathbf{g}}_{\mathrm{opt}}=\alpha \mathbf{g}_{1}$ since $\alpha \mathbf{g}_{1}^{T} \tilde{\mathbf{X}} \hat{\mathbf{W}} \hat{\mathbf{W}}^{T} \tilde{\mathbf{X}}^{T} \mathbf{g}_{1} \alpha=0$, where $\alpha=\left(\mathbf{g}_{1}^{T} C_{\mathbf{X}} \mathbf{g}_{1}\right)^{1 / 2}$. From the definitions of $\hat{\mathbf{X}}, \chi, \mathbf{W}$ and $C_{\mathbf{X}}$, we obtain the following relation:

$$
\begin{aligned}
& \hat{\mathbf{X}} \hat{\mathbf{X}}^{T}+\chi \mathbf{W} \mathbf{W}^{T} \chi^{T}=C_{\mathbf{X}} \\
\Longrightarrow & \mathbf{g}^{T} \hat{\mathbf{X}} \hat{\mathbf{X}}^{T} \mathbf{g}+\mathbf{g}^{T} \chi \mathbf{W} \mathbf{W}^{T} \chi^{T} \mathbf{g}=\mathbf{g}^{T} C_{\mathbf{X}} \mathbf{g} \\
\Longrightarrow & \mathbf{g}^{T} \hat{\mathbf{X}} \hat{\mathbf{X}}^{T} \mathbf{g}=\mathbf{g}^{T} C_{\mathbf{X}} \mathbf{g}-\mathbf{g}^{T} \chi \mathbf{W} \mathbf{W}^{T} \chi^{T} \mathbf{g} .
\end{aligned}
$$

Thus

$$
\begin{gathered}
\min _{\mathbf{g}^{T} C_{\mathbf{X g}=1}} \mathbf{g}^{T} \hat{\mathbf{X}} \hat{\mathbf{X}}^{T} \mathbf{g}=\min _{\mathbf{g}^{T} C_{\mathbf{X g}}=1}\left(\mathbf{g}^{T} C_{\mathbf{X}} \mathbf{g}-\right. \\
\left.\mathbf{g}^{T} \chi \mathbf{W} \mathbf{W}^{T} \chi^{T} \mathbf{g}\right) \\
\Longrightarrow \min _{\mathbf{g}^{T} C_{\mathbf{X g}}=1} \mathbf{g}^{T} \hat{\mathbf{X}} \hat{\mathbf{X}}^{T} \mathbf{g}=1-\max _{\mathbf{g}^{T} C_{\mathbf{X}}=1} \mathbf{g}^{T} \chi \mathbf{W} \mathbf{W}^{T} \chi^{T} \mathbf{g} .
\end{gathered}
$$

Therefore, $\hat{\mathrm{g}}_{\text {opt }}$ in (17) is equal to $\tilde{\mathrm{g}}_{\text {opt }}$ in (12). That is, if we put different weights on the columns of $\widetilde{\mathbf{X}}$ and use $\mathbf{g}^{T} C_{\mathbf{X}} \mathbf{g}=1$ as the constraint in the algorithm of Giannakis et al. , the restored image is the same as the one using the second algorithm.

\section{COMPUTER Simulations}

In Section IV, we proposed two multichannel blind image restoration algorithms based on the developments in Section III. The relation between these two algorithms and their connection to the direct restoration filter estimation algorithm were derived. The implementation issues and limitations of these two algorithms were also studied. In this chapter, we conduct computer simulations to demonstrate the second algorithm.

In all the simulations, we use zero-mean white additive Gaussian noise. Recalling that $e_{p_{1}, p_{2}}$ are noise terms and $x_{p_{1}, p_{2}}$ are blurred images at spatial position $\left(p_{1}, p_{2}\right)$, the signal-to-noise ratio (SNR) is given by

$$
\mathrm{SNR}=10 \log \frac{\sum_{i=1}^{m} \sum_{p_{1}=l_{1}-1}^{n_{1}-1} \sum_{p_{2}=l_{2}-1}^{n_{2}-1}\left(x_{p_{1}, p_{2}}^{(i)}\right)^{2}}{\sum_{i=1}^{m} \sum_{p_{1}=l_{1}-1}^{n_{1}-1} \sum_{p_{2}=l_{2}-1}^{n_{2}-1}\left(e_{p_{1}, p_{2}}^{(i)}\right)^{2}} \mathrm{~dB} .
$$

The images we use to conduct the simulations are given in Fig. 2.

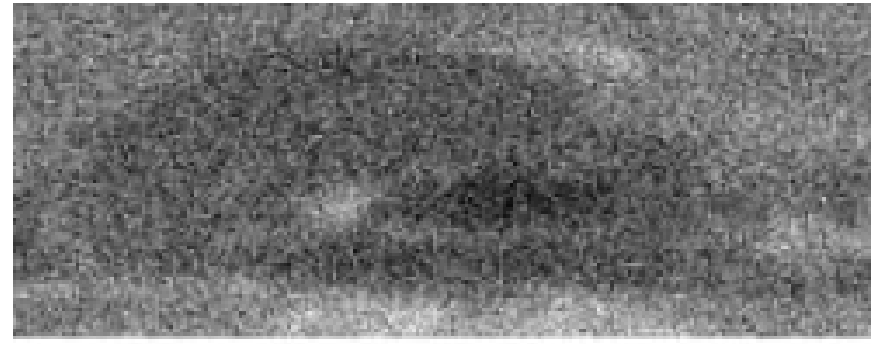

(a)

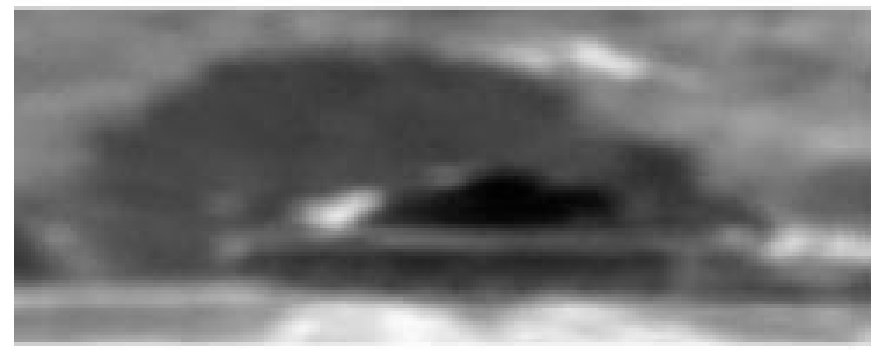

(b)

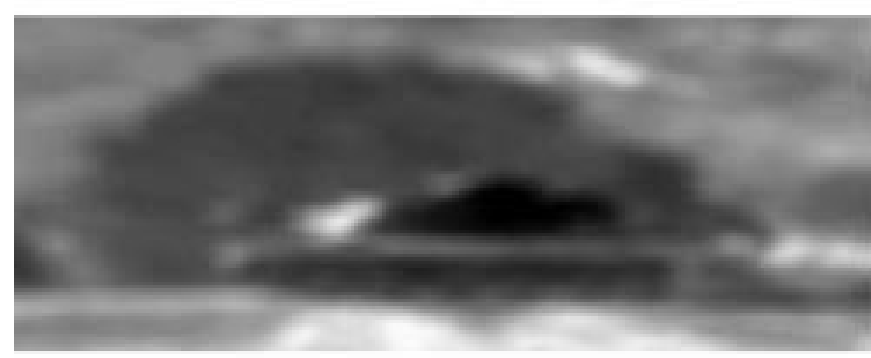

(c)

Fig. 4. Blurred images in the noisy cases: (a) $\mathrm{SNR}=10 \mathrm{~dB}$, (b) SNR $=$ $40 \mathrm{~dB}$ and (c) $\mathrm{SNR}=60 \mathrm{~dB}$.

First, we use the four blurs shown in Fig. 3 to obtain the blurred images in each case. Every blur function is a Gaussian filter with perturbation randomly generated with normal distribution. The noise is added and the SNR is 10,40 , and $60 \mathrm{~dB}$ for each case. We choose $k_{1}=4$ and $k_{2}=6$.

$$
\begin{aligned}
\underline{\mathbf{W}}_{p_{1}}^{\left(q_{2}\right)}= & {\left[\begin{array}{cccc}
w_{p_{1}, q_{2}}^{2} & 0 & \cdots & 0 \\
0 & w_{p_{1}, q_{2}+1}^{2} & \ddots & 0 \\
\vdots & \ddots & \ddots & \ddots \\
0 & \cdots & 0 & w_{p_{1}, n_{2}-r_{2}-1}^{2}
\end{array}\right] } \\
\underline{\mathbf{W}}^{\left(q_{1}, q_{2}\right)}= & {\left[\begin{array}{cccc}
\underline{\mathbf{W}}_{q_{1}}^{\left(q_{2}\right)} & 0 & \cdots & 0 \\
0 & \underline{\mathbf{W}}_{q_{1}+1}^{\left(q_{2}\right)} & \ddots & 0 \\
\vdots & \ddots & \ddots & \ddots \\
0 & \cdots & 0 & \underline{\mathbf{W}}_{n_{1}-r_{1}-1}^{\left(q_{2}\right)}
\end{array}\right] } \\
\hat{\mathbf{W}}_{p_{1}, p_{2}}= & {\left[\mathbf{W}^{(0,1)}, \ldots, \underline{\mathbf{W}}^{\left(0, r_{2}-p_{2}\right)}, \underline{\mathbf{W}}^{(1,0)}, \ldots, \mathbf{W}^{\left(r_{1}-p_{1}, r_{2}-p_{2}\right)}\right]^{T} } \\
\hat{\mathbf{W}} & =\left[\hat{\mathbf{W}}_{0,0}^{T}, \ldots, \hat{\mathbf{W}}_{0, r_{2}}^{T}, \hat{\mathbf{W}}_{1,0}^{T}, \ldots, \hat{\mathbf{W}}_{r_{1}, r_{2}-1}^{T}\right]^{T}
\end{aligned}
$$




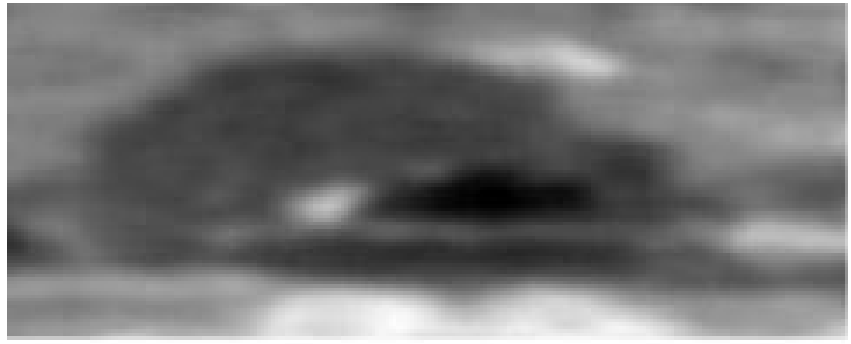

(a)

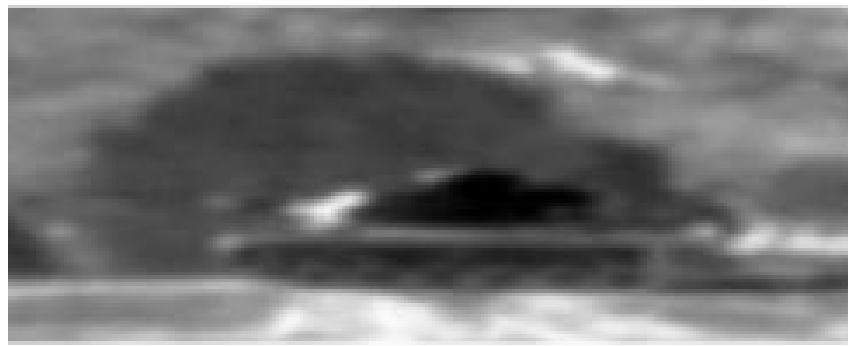

(b)

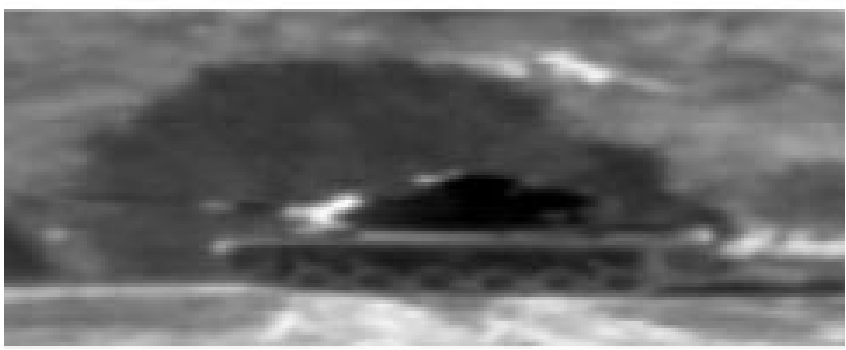

(c)

Fig. 5. Restored images in the different noisy cases: (a) SNR $=10 \mathrm{~dB}$, (b) $\mathrm{SNR}=40 \mathrm{~dB}$ and $(\mathrm{c}) \mathrm{SNR}=60 \mathrm{~dB}$

Fig. 4 shows one of the noisy blurred images in each case. The restored images are given in Fig. 5. We can see that the higher the SNR is, the clearer the restored image is. Furthermore, no noise amplification appears in the low SNR cases. The restored images in the low SNR cases are just as unclear as the blurred image since solving the optimization problem tends to smooth the noise in the low SNR cases and this smoothing causes the image blurred.

Second, we use four $1 \times 1$ blur functions to obtain four "blurred" images. That is, each of these blurred images is equal to the original image, up to a scalar multiple. The noise is then added to the blurred images with $\mathrm{SNR}=10 \mathrm{~dB}$. One of the noisy blurred images is shown in Fig. 6(a). Fig. 6(b) and (c) are the restored images obtained by the second algorithm and averaging these four noisy blurred images respectively. These two restored images are quite similar. Again, there is no noise amplification using the second algorithm.

\section{CONCLUSION}

We solved a nullspace-based multichannel blind image restoration problem using matrix operations before. Actually, this problem can be regarded as an constrained optimization problem. By using different constraints, different optimization

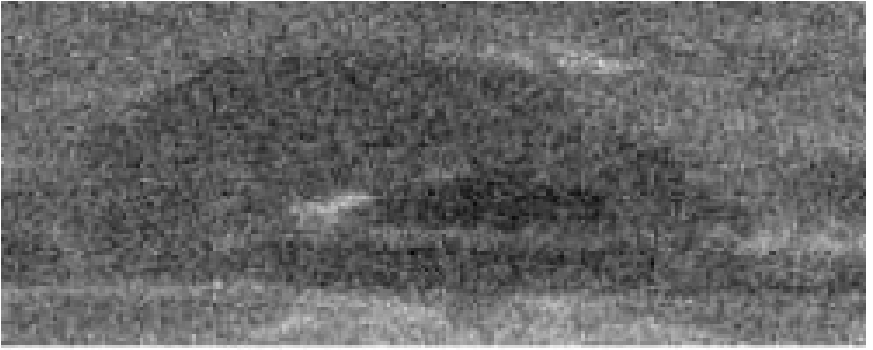

(a)

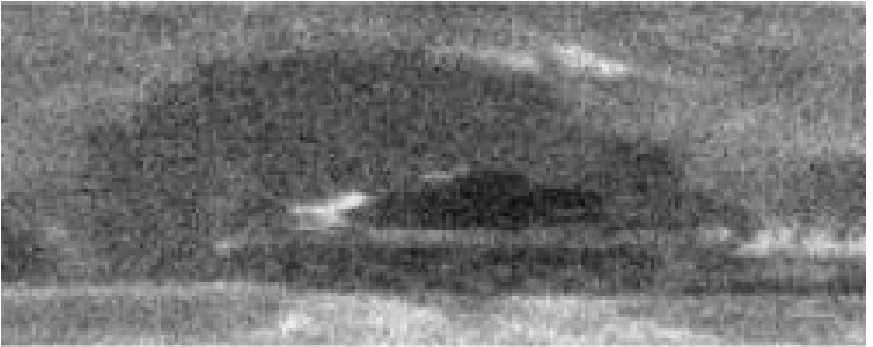

(b)

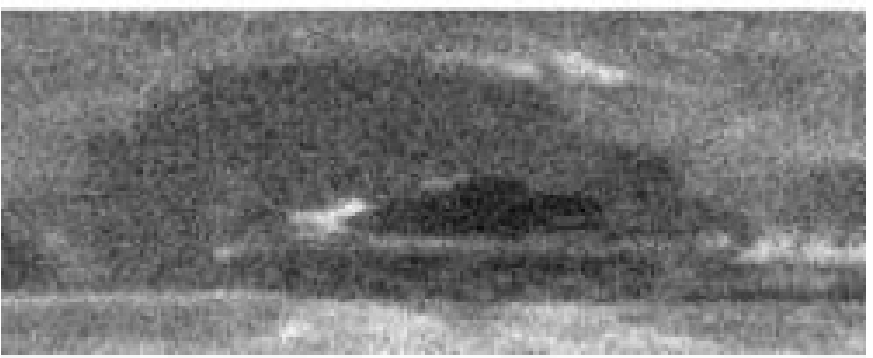

(c)

Fig. 6. (a) One of the noisy images "blurred" by a scalar, where SNR $=10 \mathrm{~dB}$, (b) the restored image using the second algorithm, and (c) the restored image by averaging the noisy images.

problems are formulated. One of them can also be solved by matrix operations. A different nullspace-based multichannel blind image restoration algorithm is obtained.

The formulation of the different optimization problems implies a new column-space-based algorithm. The restored images by this new algorithm and by the different nullspace-based one are the same. This new algorithm has the same advantage as the nullspace-based one, such as exact restoration and no noise amplification. Furthermore, the new algorithm requires less computational complexity than the nullspace-based one. Actually, under some mild conditions, the complexity of this new algorithm is equal to FFT complexity.

Another eigenstructure-based direct multichannel blind image restoration algorithm is direct deconvolver estimation. We also formulate it as an optimization problem. We then make a connection between it and the new algorithm. By using a different constraint and putting some weighting on the objective function of the optimization problem, the direct deconvolver estimation approach is equivalent to the new algorithm.

We have thoroughly studied eigenstructure-based techniques for direct multichannel blind image restoration. The LTI FIR model was used and the size of the blur channels are assumed 
in these techniques. These limitations should be removed in the future. Further, we should move to solve nonlinear and/or nontime-invariant problems.

\section{APPENDIX \\ Proofs of Lemmas IN SECTION III}

In this section, $t$ is an $n_{1} \times n_{2}$ image. $\mathbf{T}$ and $\mathbf{t}$ are the corresponding matrices of $t$ as $s$ is to $\mathbf{S}$ and $\mathbf{s}$, respectively. Furthermore, $\mathbf{t}_{p_{1}, p_{2}}^{T}$ is the $\left(p_{1} r_{2}+p_{2}\right)$ th row of $\mathbf{T}$.

\section{A. Proof of Lemma 1}

If $t$ is a solution of (7), then

$$
\begin{aligned}
& \arg \min _{y}\|\mathbf{Y N}\{\mathbf{X}\}\|^{2} \\
& \text { subject to }\|\mathbf{Y}\|^{2}=1 .
\end{aligned}
$$

That is

$$
\sum_{p_{1}=0}^{r_{1}} \sum_{p_{2}=0}^{r_{2}}\left\|\mathcal{N}\{\mathbf{X}\} \mathbf{t}_{p_{1}, p_{2}}\right\|^{2}=0
$$

This implies that

$$
\begin{aligned}
\left\|\mathcal{N}\{\mathbf{X}\} \mathbf{t}_{p_{1}, p_{2}}\right\|^{2}=0, & \text { for } p_{1}=0,1, \ldots, r_{1} \text { and } \\
& p_{1}=0,1, \ldots, r_{2} .
\end{aligned}
$$

Therefore, the null space of $\mathbf{T}$ must include $\mathcal{N}\{\mathbf{X}\}=\mathcal{N}\{\mathbf{S}\}$, which means that $\mathcal{R}\left\{\mathbf{T}^{T}\right\} \subset \mathcal{R}\left\{\mathbf{S}^{T}\right\}$. From [26], $t=\alpha s$, where $\alpha$ is a nonzero scalar.

\section{B. Proof of Lemma 2}

Let

$$
\begin{aligned}
\tilde{\mathbf{W}}_{p_{1}}^{\left(q_{2}\right)} & =\left[\begin{array}{cccc}
w_{p_{1}, q_{2}} & 0 & \cdots & 0 \\
0 & w_{p_{1}, q_{2}+1} & \ddots & 0 \\
\vdots & \ddots & \ddots & \ddots \\
0 & \cdots & 0 & w_{p_{1}, n_{2}-r_{2}-1+q_{2}}
\end{array}\right] \\
\tilde{\mathbf{W}}^{\left(q_{1}, q_{2}\right)} & =\left[\begin{array}{cccc}
\tilde{\mathbf{W}}_{q_{1}}^{\left(q_{2}\right)} & 0 & \cdots & 0 \\
0 & \tilde{\mathbf{W}}_{q_{1}+1}^{\left(q_{2}\right)} & \ddots & 0 \\
\vdots & \ddots & \ddots & \ddots \\
0 & \cdots & 0 & \tilde{\mathbf{W}}_{n_{1}-r_{1}-1+q_{1}}^{\left(q_{2}\right)}
\end{array}\right] .
\end{aligned}
$$

Notice that the matrix $\tilde{\mathbf{W}}^{\left(q_{1}, q_{2}\right)}$ is an $\left(n_{1}-r_{1}\right)\left(n_{2}-r_{2}\right) \times\left(n_{1}-\right.$ $\left.r_{1}\right)\left(n_{2}-r_{2}\right)$ matrix. Since

$$
\|\tilde{\mathbf{V}} \mathbf{y}\|^{2}=\sum_{p_{1}=0}^{r_{1}} \sum_{p_{2}=0}^{r_{2}}\left\|\mathcal{N}\{\mathbf{X}\} \tilde{\mathbf{W}}^{\left(p_{1}, p_{2}\right)} \mathbf{y}_{p_{1}, p_{2}}\right\|^{2}
$$

the null vector of $\tilde{\mathbf{V}}$ is the unique solution of the following optimization problem

$$
\begin{gathered}
\arg \min _{y} \sum_{p_{1}=0}^{r_{1}} \sum_{p_{2}=0}^{r_{2}}\left\|\mathcal{N}\{\mathbf{X}\} \tilde{\mathbf{W}}^{\left(p_{1}, p_{2}\right)} \mathbf{y}_{p_{1}, p_{2}}\right\|^{2}, \\
\text { given }\|\mathbf{y}\|^{2}=1 .
\end{gathered}
$$

Let $\tilde{y}_{p_{1}, p_{2}}=y_{p_{1}, p_{2}} w_{p_{1}, p_{2}}$ for $p_{1}=0,1, \ldots, n_{1}-1$ and $p_{2}=$ $0,1, \ldots, n_{2}-1$. (18) can be rewritten as

$$
\begin{aligned}
\arg \min _{\tilde{y}} \sum_{p_{1}=0}^{r_{1}} \sum_{p_{2}=0}^{r_{2}}\left\|\mathcal{N}\{\mathbf{X}\} \tilde{\mathbf{y}}_{p_{1}, p_{2}}\right\|^{2}, \\
\quad \text { given } \sum_{p_{1}=0}^{r_{1}} \sum_{p_{2}=0}^{r_{2}}\left\|\tilde{\mathbf{y}}_{p_{1}, p_{2}}\right\|^{2}=1 .
\end{aligned}
$$

Comparing (19) with (7), the solutions of these two optimization problems are the same. As a result, the product of $\mathbf{W}$ and the null vector of $\tilde{\mathbf{V}}$ is the same as the solution of (7).

\section{Proof of Lemma 3}

For any $t$

$$
\begin{aligned}
& \left\|\mathcal{R}\left\{\mathbf{X}^{\mathcal{T}}\right\} \mathbf{t}_{p_{1}, p_{2}}\right\|^{2} \leq\left\|\mathbf{t}_{p_{1}, p_{2}}\right\|^{2}, \\
& \text { for } p_{1}=0,1, \ldots, r_{1}, p_{2}=0,1, \ldots, r_{2} .
\end{aligned}
$$

If $t$ is a solution of (8), then

$$
\begin{array}{r}
\sum_{p_{1}=0}^{r_{1}} \sum_{p_{2}=0}^{r_{2}}\left\|\mathbf{t}_{p_{1}, p_{2}}\right\|^{2}=\sum_{p_{1}=0}^{r_{1}} \sum_{p_{2}=0}^{r_{2}}\left\|\mathbf{s}_{p_{1}, p_{2}}\right\|^{2} \\
\sum_{p_{1}=0}^{r_{1}} \sum_{p_{2}=0}^{r_{2}}\left\|\mathcal{R}\left\{\mathbf{X}^{T}\right\} \mathbf{t}_{p_{1}, p_{2}}\right\|^{2}=\sum_{p_{1}=0}^{r_{1}} \sum_{p_{2}=0}^{r_{2}}\left\|\mathbf{s}_{p_{1}, p_{2}}\right\|^{2} .
\end{array}
$$

From (20), this implies

$$
\begin{aligned}
& \left\|\mathcal{R}\left\{\mathbf{X}^{T}\right\} \mathbf{t}_{p_{1}, p_{2}}\right\|^{2}=\left\|\mathbf{t}_{p_{1}, p_{2}}\right\|^{2}, \\
& p_{1}=0,1, \ldots, r_{1} \quad p_{2}=0,2, \ldots, r_{2} .
\end{aligned}
$$

Therefore, $\mathbf{t}_{p_{1}, p_{2}} \in \mathcal{R}\left\{\mathbf{X}^{T}\right\}=\mathcal{R}\left\{\mathbf{S}^{T}\right\}$ for $p_{1}=0,1, \ldots, r_{1}$ and $p_{2}=0,1, \ldots, r_{2}$. This means that $\mathcal{R}\left\{\mathbf{T}^{T}\right\} \subset \mathcal{R}\left\{\mathbf{S}^{T}\right\}$. From [26], $t=\alpha s$, where $\alpha$ is a nonzero scalar.

\section{Proof of Lemma 4}

The definitions of $\tilde{\mathbf{W}}^{\left(p_{1}, p_{2}\right)}$ and $\tilde{y}_{p_{1}, p_{2}}$ are the same as in Lemma 2. Since

$$
\|\tilde{\mathbf{U}} \mathbf{y}\|^{2}=\sum_{p_{1}=0}^{r_{1}} \sum_{p_{2}=0}^{r_{2}}\left\|\mathbf{R}\left\{\mathbf{X}^{T}\right\} \tilde{\mathbf{W}}^{\left(p_{1}, p_{2}\right)} \mathbf{y}_{p_{1}, p_{2}}\right\|^{2}
$$

the right singular vector corresponding to the largest singular value of $\tilde{\mathbf{U}}$ is the unique solution of the following optimization problem:

$\arg \min _{y} \sum_{p_{1}=0}^{r_{1}} \sum_{p_{2}=0}^{r_{2}}\left\|\mathbf{R}\left\{\mathbf{X}^{T}\right\} \tilde{\mathbf{W}}^{\left(p_{1}, p_{2}\right)} \mathbf{y}_{p_{1}, p_{2}}\right\|^{2}$, given $\|\mathbf{y}\|^{2}=1$.

Using the definition of $\tilde{y}_{p_{1}, p_{2}}$, (21) can be rewritten as

$$
\begin{aligned}
\arg \min _{\tilde{y}} \sum_{p_{1}=0}^{r_{1}} \sum_{p_{2}=0}^{r_{2}}\left\|\mathbf{R}\left\{\mathbf{X}^{T}\right\} \tilde{\mathbf{y}}_{p_{1}, p_{2}}\right\|^{2}, \\
\quad \text { given } \sum_{p_{1}=0}^{r_{1}} \sum_{p_{2}=0}^{r_{2}}\left\|\tilde{\mathbf{y}}_{p_{1}, p_{2}}\right\|^{2}=1 .
\end{aligned}
$$

Comparing (22) with (8), the solutions of these two optimization problems are the same. As a result, the product of $\mathbf{W}$ and the 
right singular vector corresponding to the largest singular value of $\tilde{\mathbf{U}}$ is the same as the solution of (8).

\section{REFERENCES}

[1] M. R. Banham and A. K. Katsaggelos, "Digital image restoration," IEEE Signal Processing Mag., vol. 14, pp. 24-41, Mar. 1997.

[2] A. K. Katsaggelos, Ed., Digital Image Restoration. Berlin, Germany: Springer-Verlag, 1991.

[3] D. Kundur and D. Hatzinakos, "Blind image deconvolution," IEEE Signal Processing Mag., vol. 13, pp. 43-64, May 1996.

[4] - "Blind image deconvolution revisited," IEEE Signal Processing Mag., vol. 13, pp. 61-63, Nov. 1996.

[5] R. K. Ward, "Restoration of differently blurred versions of an image with measurement errors in the PSF's," IEEE Trans. Image Processing, vol. 2, pp. 369-381, July 1993.

[6] T. J. Schulz, "Multiframe blind deconvolution of astronomical images," J. Opt. Soc. Amer. A., vol. 10, pp. 1064-1073, May 1993.

[7] N. Miura and N. Baba, "Extended-object reconstruction with sequential use of the iterative blind deconvolution method," Opt. Commun., vol. 89, pp. 375-379, May 1992.

[8] L. Tong, G. Xu, and T. Kailath, "Blind identification and equalization based on second-order statistics: A time domain approach," IEEE Trans. Inform. Theory, vol. 40, pp. 340-349, Mar. 1994.

[9] L. Tong, G. Xu, B. Hassibi, and T. Kailath, "Blind channel identification based on second-order statistics: A frequency-domain approach," IEEE Trans. Inform. Theory, vol. 41, pp. 329-334, Jan. 1995.

[10] H. H. Zeng and L. Tong, "Blind channel estimation using the secondorder statistics: Asymptotic performance and limitations," IEEE Trans. Signal Processing, vol. 45, pp. 2060-2071, Aug. 1997.

[11] _ "Blind channel estimation using the second-order statistics: Algorithms," IEEE Trans. Signal Processing, vol. 45, pp. 1919-1930, Aug. 1997.

[12] G. B. Giannakis and S. D. Halford, "Asymptotically optimal blind fractionally-spaced channel estimation and performance analysis," IEEE Trans. Signal Processing, vol. 45, pp. 1815-1830, July 1997.

[13] D. T. M. Slock, "Blind fractionally-spaced equalization, perfect-reconstruction filter banks and multichannel linear prediction," in Proc. IEEE Int. Conf. Acoust., Speech, Signal Processing, vol. 4, Adelaide, Australia, Apr. 1994, pp. 585-588.

[14] E. Moulines, P. Duhamel, J. F. Cardoso, and S. Mayrargue, "Subspace methods for the blind identification of multichannel FIR filters," IEEE Trans. Signal Processing, vol. 43, pp. 516-525, Feb. 1995.

[15] G. Xu, H. Liu, L. Tong, and T. Kailath, "A least-squares approach to blind channel identification," IEEE Trans. Signal Processing, vol. 43, pp. 2982-2993, Dec. 1995

[16] M. İ. Gürelli and C. L. Nikias, "EVAM : An eigenvector-based algorithm for multichannel blind deconvolution of input colored signals," IEEE Trans. Signal Processing, vol. 43, pp. 134-149, Jan. 1995.

[17] C. A. Berenstein, R. Gay, A. Vidras, and A. Yger, Residue Currents and Bezout Identities. Berlin, Germany: Birkhäuser Verlag, 1993.

[18] D. T. M. Slock and C. B. Papadias, "Further results on blind identification and equalization of multiple FIR channels," in Proc. IEEE Int. Conf. Acoust., Speech, Signal Processing, vol. 3, Detroit, MI, May 1995, pp. 1964-1967.

[19] D. Gesbert, P. Duhamel, and S. Mayrargue, "On-line blind multichannel equalization based on mutually referenced filters," IEEE Trans. Signal Processing, vol. 45, pp. 2307-2317, Sept. 1997.

[20] G. Harikumar and Y. Bresler, "Exact image deconvolution from multiple FIR blurs," IEEE Trans. Image Processing, vol. 8, pp. 846-862, June 1999.

[21] — - "Perfect blind restoration of images blurred by multiple filters: Theory and efficient algorithms," IEEE Trans. Image Processing, vol. 8, pp. 202-219, Feb. 1999

[22] G. B. Giannakis and R. W. Heath Jr, "Blind identification of multichannel FIR blur and perfect image restoration," IEEE Trans. Image Processing, vol. 9, pp. 1877-1896, Nov. 2000.

[23] H. Liu and G. Xu, "Closed-form blind symbol estimation in digital communications," IEEE Trans. Signal Processing, vol. 43, pp. 2714-2723, Nov. 1995.
[24] A. J. van der Veen, S. Talwar, and A. Paulraj, "A subspace approach to blind space-time signal processing for wireless communication systems," IEEE Trans. Signal Processing, vol. 45, pp. 173-190, Jan. 1997.

[25] D. Gesbert, A. J. van der Veen, and A. Paulraj, "On the equivalence of blind equalizers based on the MRE and subspace intersections," IEEE Trans. Signal Processing, vol. 47, pp. 856-859, Mar. 1999.

[26] H. T. Pai and A. C. Bovik, "Exact multichannel blind image restoration," Signal Processing Lett., vol. 4, pp. 217-220, Aug. 1997.

[27] H. T. Pai, A. C. Bovik, and B. L. Evans, "Multichannel blind image restoration," Elektrik, vol. 5, no. 1, pp. 79-97, 1998.

[28] H. T. Pai, J. W. Havlicek, and A. C. Bovik, "Generically sufficient conditions for exact multichannel blind image restoration," in Proc. IEEE Int. Conf. Acoust., Speech, Signal Processing, vol. 2, Seattle, WA, Oct. 1998, pp. 2861-2864.

[29] G. Strang, Linear Algebra and its Applications, 3 ed. San Diego, CA: Harcourt Brace Jovanovich, 1988.

[30] E. Andersion, Z. Bai, C. Bischof, J. Demmel, J. Dongarra, J. Du Croz, A. Greenbaum, S. Hammarling, A. McKenney, S. Ostrouchov, and D. Sorensen, LAPACK Users' Guide, 2 ed. Philadelphia, PA: SIAM, 1995.

[31] D. S. Watkins, Fundamentals of Matrix Computations. New York: Wiley, 1991.

[32] H. H. Zeng and L. Tong, "Connections between the least-squares and the subspace approaches to blind channel estimation," IEEE Trans. Signal Processing, vol. 44, pp. 1993-1996, June 1996.

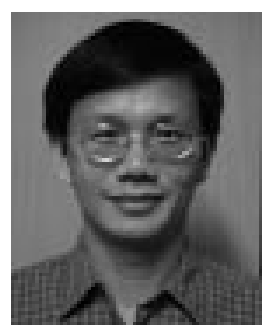

Hung-Ta Pai (M'99) was born in Taichung, Taiwan, R.O.C., in 1970. He received the B.S. degree from National Tsing Hua University, Taiwan, in 1992 and the M.S. and Ph.D. degrees from the University of Texas at Austin in 1996 and 1999, respectively, all in electrical engineering.

From 1992 to 1994, he served as an Officer in the Army. Since 1999, he has been a Senior Engineer at Silicon Integrated Systems Corp., Taiwan. His interests include digital signal processing and digital communications.

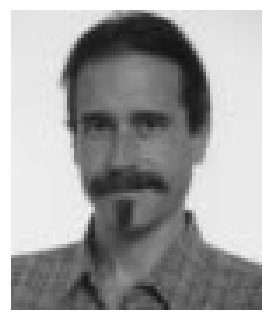

Alan Conrad Bovik (S'80-M'81-SM'89-F'96) received the B.S., M.S., and Ph.D. degrees in electrical and computer engineering in 1980, 1982, and 1984, respectively, all from the University of Illinois, Urbana-Champaign.

$\mathrm{He}$ is currently the Robert Parker Centennial Endowed Professor in the Department of Electrical and Computer Engineering, University of Texas at Austin. During the Spring of 1992, he held a visiting position in the Division of Applied Sciences, Harvard University, Cambridge, MA. His current research interests include digital video, image processing, computer vision, wavelets, three-dimensional microscopy, and computational aspects of biological visual perception. He has published more than 300 technical articles in these areas and holds two U.S. patents. He is the editor/author of the Handbook of Image and Video Processing (New York: Academic, 2000).

Dr. Bovik was named Distinguished Lecturer of the IEEE Signal Processing Society in 2000, received the IEEE Signal Processing Society Meritorious Service Award in 1998, the IEEE Third Millennium Medal in 2000, the University of Texas Engineering Foundation Halliburton Award, and is a two-time Honorable Mention winner of the International Pattern Recognition Society Award for Outstanding Contribution (1988 and 1993). He has been involved in numerous professional society activities, including his current ones: Editor-in-Chief of the IEEE TRANSACTIONS ON IMAGE PROCESSING and Editorial Board Member of the PROCEEDINGS OF THE IEEE. He also serves on the editorial boards of several other technical journals. He was the Founding General Chairman of the $1 s t$ IEEE International Conference on Image Processing, held in Austin in 1994. $\mathrm{He}$ is a registered Professional Engineer in the State of Texas and is a frequent consultant to industry and academic institutions. 